\title{
Revisiting long-standing puzzles of the Milky Way: the Sun and its vicinity as typical outer disk chemical evolution
}

\author{
M. Haywood ${ }^{1,3}$, O. Snaith ${ }^{2}$, M. D. Lehnert ${ }^{3}$, P. Di Matteo ${ }^{1,3}$, and S. Khoperskov ${ }^{1,4}$ \\ 1 GEPI, Observatoire de Paris, PSL Research University, CNRS, Sorbonne Paris Cité, 5 place Jules Janssen, 92190 Meudon, France \\ e-mail: misha.haywood@obspm. fr \\ 2 School of Physics, Korea Institute for Advanced Study, 85 Hoegiro, Dongdaemun-gu, Seoul 02455, Republic of Korea \\ 3 Sorbonne Université, CNRS UMR 7095, Institut d'Astrophysique de Paris, 98bis bd Arago, 75014 Paris, France \\ ${ }^{4}$ Max Planck Institute for Extraterrestrial Physics, 85741 Garching, Germany
}

Received 29 August 2018 / Accepted 26 January 2019

\begin{abstract}
We present a scenario of the chemical enrichment of the solar neighborhood that solves the G-dwarf problem by taking into account constraints on a larger scale. We argue that the Milky Way disk within $10 \mathrm{kpc}$ has been enriched to solar metallicity by a massive stellar population: the thick disk, which itself formed from a massive turbulent gaseous disk. While the inner disk, $R \lesssim 6 \mathrm{kpc}$, continued this enrichment after a quenching phase $(7-10 \mathrm{Gyr})$, at larger distances radial flows of gas diluted the metals left by the thick disk formation at a time we estimate to be $7-8 \mathrm{Gyr}$ ago, thus partitioning the disk into an inner and outer region characterized by different chemical evolutions. The key new consideration is that the pre-enrichment provided by the thick disk is not related to the mass fraction of this stellar population at the solar radius, as is classically assumed in inside-out scenarios, but is actually related to the formation of the entire massive thick disk, due to the vigorous gas phase mixing that occurred during its formation. Hence, the fact that this population represents only $15-25 \%$ of the local stellar surface density today, or $5-10 \%$ of the local volume density, is irrelevant for "solving" the G-dwarf problem. The only condition for this scenario to work is that the thick disk was formed from a turbulent gaseous disk that permitted a homogeneous - not radially dependent - distribution of metals, allowing the solar ring to be enriched to solar metallicity. At the solar radius, the gas flowing from the outer disk combined with the solar metallicity gas left over from thick disk formation, providing the fuel necessary to form the thin disk at the correct metallicity to solve the G-dwarf problem. Chemical evolution at $R>6 \mathrm{kpc}$, and in particular beyond the solar radius, can be reproduced with the same scheme. We suggest that the dilution, occurring at the fringe of the thick disk, was possibly triggered by the formation of the bar and the establishment of the outer Lindblad resonance (OLR), enabling the inflow of metal poorer gas from the outer disk to $R \sim 6 \mathrm{kpc}$, presumably the position of the OLR at this epoch, and at the same time isolating the inner disk from external influence. These results imply that the local metallicity distribution is not connected to the gas accretion history of the Milky Way. Finally, we argue that the Sun is the result of the evolution typical of stars in the disk beyond $\sim 6 \mathrm{kpc}$ (i.e., also undergoing dilution), and has none of the characteristics of inner disk stars.
\end{abstract}

Key words. Galaxy: abundances - Galaxy: disk - Galaxy: evolution

\section{Introduction}

Cold gas accretion (Dekel \& Birnboim 2006; Woods et al. 2014; Tillson et al. 2015), which in the last ten years has become the new paradigm describing how galaxies acquire their gas, predicts that considerable gas accretion occurs along a few dark matter filaments (Birnboim \& Dekel 2003; Kereš et al. 2005, 2009; Ocvirk et al. 2008; Agertz et al. 2009; Cornuault et al. 2018), driving large amounts of fuel in the inner parts of galaxies, permitting the early buildup of large disks (Genzel et al. 2006, 2017; Toft et al. 2017), and possibly leading to the formation of large gas reservoirs (Davé et al. 2012; Papovich et al. 2011; Hopkins et al. 2014; Suess et al. 2017). Observations show that disks are indeed already massive at $z \sim 1.5$, with roughly half their stellar mass already in place for Milky Way-mass galaxies (Muzzin et al. 2013; van Dokkum et al. 2013; Patel et al. 2013; Papovich et al. 2015), and perhaps as much mass in molecular gas (Tacconi et al. 2013; Dessauges-Zavadsky et al. 2015; Saintonge et al. 2013; Papovich et al. 2016).

Our Galaxy is compatible with this overall picture. In Snaith et al. (2015), and Haywood et al. (2016, 2018) we show that the main chemical properties of the inner Milky Way, $R \lesssim$
$6 \mathrm{kpc}$ (disk and bulge), are compatible with a scheme where the gas has been accreted early by our Galaxy. The substantial number of low-metallicity dwarf stars that exist in the inner Milky Way (see the metallicity distribution function, MDF, in Anders et al. 2014 or Haywood et al. 2018) is in agreement with the predictions of a closed-box model where the star formation history ( $\mathrm{SFH}$ ) has two predominate phases, one corresponding to the growth of the thick disk and the other to the growth of the thin disk. In Snaith et al. $(2014,2015)$ we show that half of the disk stellar mass is due to the thick disk. The thick disk stars that we see in the solar vicinity are therefore the "tip of the iceberg" of a significantly more massive population, which, having a short scale length (Bensby et al. 2011; Cheng et al. 2012; Bovy et al. 2012a), is mainly confined in the inner Milky Way. In standard chemical evolution models (e.g., Chiappini et al. 1997; Colavitti et al. 2009; Marcon-Uchida et al. 2010; Minchev et al. 2014; Kubryk et al. 2015), the presence of such a huge stellar population has a limited consequence on the evolution at the solar circle because the Galactic disk is conceived as being made of independent rings whose evolution is usually not connected to the others in order to reproduce the inside-out paradigm of galaxy evolution. Hence, in these models the chemical 
evolution of the thick disk seen at the solar vicinity is simply dictated by the evolution of the fractional mass of this population at the solar vicinity, while the evolution of the stellar populations in the inner disk has no impact. This independence with radius of standard chemical evolution models has been mitigated in the last decade by allowing for an unconstrained amount of radial migration of the stars, in effect allowing yet other set of free parameters within models to fit the dispersion and the mode of the metallicity distributions within the Milky Way (e.g., Minchev et al. 2013; Kubryk et al. 2015; Loebman et al. 2016; Toyouchi \& Chiba 2018).

In Haywood et al. $(2013,2015,2018)$, we argued that there is good evidence that the formation of the thick disk is not insideout. This is also what is observed in APOGEE: the chemical track of alpha-rich stars in the $[\mathrm{Fe} / \mathrm{H}]-[\alpha / \mathrm{Fe}]$ plane is independent of the distance to the Galactic center (Hayden et al. 2015). We advocated that the conditions that must have prevailed in the interstellar medium (ISM) of disks at redshift greater than 2 , allowing for strong turbulence and feedback from vigorous star formation (see also Lehnert et al. 2014), must have favored large mixing of chemical species, explaining the lack of evidence of an inside-out formation of the thick disk. This has important consequences on how we see the chemical evolution of the disk at the solar vicinity, and in particular on what is known as the G-dwarf problem.

The G-dwarf problem (van den Bergh 1962; Pagel \& Patchett 1975), one of the longest standing problems in galactic astrophysics, is the recognition that local data offer no simple explanation of how the Galaxy reached the metallicity above which most stars are found at the solar vicinity (or $[\mathrm{Fe} / \mathrm{H}] \sim-0.2$ ).

If most of the gas had been in the disk at early times, thus actively forming stars (as suggested by the picture outlined above), the number of stars at $[\mathrm{Fe} / \mathrm{H}] \leq-0.2$ necessary to increase the metallicity of this large pool would have had to be a sizable fraction of the present local stellar density; instead, there are (at least in the solar vicinity) only a relatively small number of these stars. The local fraction of thick disk stars seems, on the contrary, to imply models where the disk would have been parsimoniously supplied with gas (the gas infall models, see references above), again at variance with the general picture sketched out at the beginning of this introduction.

In the present study, we explore the simple idea that if the formation of the thick disk is a global process (i.e., not inside-out), the enrichment it provides cannot be accounted for in proportion to its local mass fraction, but that it results from the chemical evolution of an entire massive population (a few $10^{10} M_{\odot}$ ) of the inner disk. In this scheme, the solar ring, although at the outskirts of the thick disk, may have been enriched by this massive stellar population of the thick disk, due to the efficient mixing within the ISM that prevailed at this epoch, thus solving the long standing G-dwarf problem. By describing the evolution of the outer disk, which includes the solar vicinity (see Sect. 5), this work complements our investigation of the evolution of the inner disk of the Galaxy, $R<6 \mathrm{kpc}$ (Haywood et al. 2018). The outline of the paper is as follows. In Sect. 2 we first start by revisiting the constraints provided by the $[\mathrm{Fe} / \mathrm{H}]-$ $[\alpha / \mathrm{Fe}]$, age- $[\alpha / \mathrm{Fe}]$ distributions of stars and the radial metallicity gradient. In Sect. 3 we explain how our new scenario is applicable to the evolution of stars at the solar ring, given the constraints advocated in the previous section. In Sect. 4 we generalize our new picture to the outer disk. In Sect. 6 we discuss some important issues. We present our conclusions in Sect. 7.

\section{Deconstructing the local chemical patterns: the $[\mathrm{Fe} / \mathrm{H}]-[\alpha / \mathrm{Fe}]$ and age- $[\alpha / \mathrm{Fe}]$ planes}

At the solar vicinity most of the stars have a metallicity $[\mathrm{Fe} / \mathrm{H}]>-0.2$. Understanding how the Milky Way ISM was locally enriched to $[\mathrm{Fe} / \mathrm{H}] \sim-0.2$ implies that we correctly interpret the two sequences that have stars below this limit of -0.2 : the low and high alpha-abundances. These two sequences are dominant in the inner and outer disks, respectively (Hayden et al. 2015).

We now review the constraints offered by these two sequences when combined with stellar ages. We also refer the reader to the study of Buder et al. (2019) and the GALAH survey.

\subsection{Inner disk sequence: a temporal sequence}

Figure 1 shows the $[\mathrm{Fe} / \mathrm{H}]-[\alpha / \mathrm{Fe}]$ and age- $[\alpha / \mathrm{Fe}]$ distributions of stars from Adibekyan et al. (2012) with ages from Haywood et al. (2013). The atmospheric parameters and chemical compositions are taken from Adibekyan et al. (2012), a sample of nearby targets observed for the purposes of searching extrasolar planets. This produced a sample of 1111 stars with temperatures, elemental abundances, stellar velocities, and associated errors. As described in Haywood et al. (2013), the selection of stars with age determinations with adequate accuracy means that we had to severely prune the original sample to only 363 stars. See Haywood et al. (2013, 2015) for detailed explanations on how the ages were derived and the expected accuracies. Since our results are not derived from stellar densities along the age- $[\alpha / \mathrm{Fe}]$ relation or in the $[\mathrm{Fe} / \mathrm{H}]-[\alpha / \mathrm{Fe}]$ plane, there should be no bias due to the volume definition of the sample. Our sample is as complete as other larger surveys within the range $-1<[\mathrm{Fe} / \mathrm{H}]<0.5$ in the $[\mathrm{Fe} / \mathrm{H}]-[\alpha / \mathrm{Fe}]$ plane.

In a recent study, Silva Aguirre et al. (2018) claims, on the basis of their asteroseimologic ages, that a tight relation between age and $[\alpha / \mathrm{Fe}]$ or $[\mathrm{Fe} / \mathrm{H}]$ does not exist during the formation of the thick disk, in contradiction with our results. However, if there are no relations between age- $[\mathrm{Fe} / \mathrm{H}]$ and age- $[\alpha / \mathrm{Fe}]$, it means that on the high- $\alpha$ sequence a star of a given low metallicity and high alpha-abundance can be younger than another star with higher metallicity and lower alpha-abundance. Because the high$\alpha$ sequence (in the $[\mathrm{Fe} / \mathrm{H}]-[\alpha / \mathrm{Fe}]$ plane) is continuous and has relatively small scatter, it would be extremely difficult to explain how the chemical evolution proceeded to maintain the underlying complexity in the age- $[\mathrm{Fe} / \mathrm{H}]-[\alpha / \mathrm{Fe}]$ space while maintaining such a tight relation in the $[\mathrm{Fe} / \mathrm{H}]-[\alpha / \mathrm{Fe}]$ plane. The natural evolution of all chemical evolution models is to decrease relative alpha-abundances while increasing metallicity with time. While heterogenous chemical evolution may exist, we see no reason why it would have conspired to give a tight high- $\alpha$ sequence. Second, $[\alpha / \mathrm{Fe}]$-kinematics and $[\alpha / \mathrm{Fe}]$-structural parameters do show conspicuous correlations: $[\alpha / \mathrm{Fe}]$ abundances correlate well on the high- $\alpha$ sequence with an increase in the velocity dispersions or orbital parameters (Haywood et al. 2013; Bensby et al. 2014). We also do not see how these correlations could exist at all if $[\alpha / \mathrm{Fe}]$ was not correlated with age, or was only loosely correlated. Likewise, the increase in scale heights with $[\alpha / \mathrm{Fe}]$ abundance (Bovy et al. 2012a) would simply not exist without a relation linking age and $[\alpha / \mathrm{Fe}]$. Moreover, given that $[\alpha / \mathrm{Fe}]$ derived from SEGUE are based on low signal-to-noise, lowdispersion spectra, the underlying correlation between age and $[\alpha / \mathrm{Fe}]$ abundance must be rather strong to still be visible in the data (Bovy et al. 2012a). Our final argument comes from 

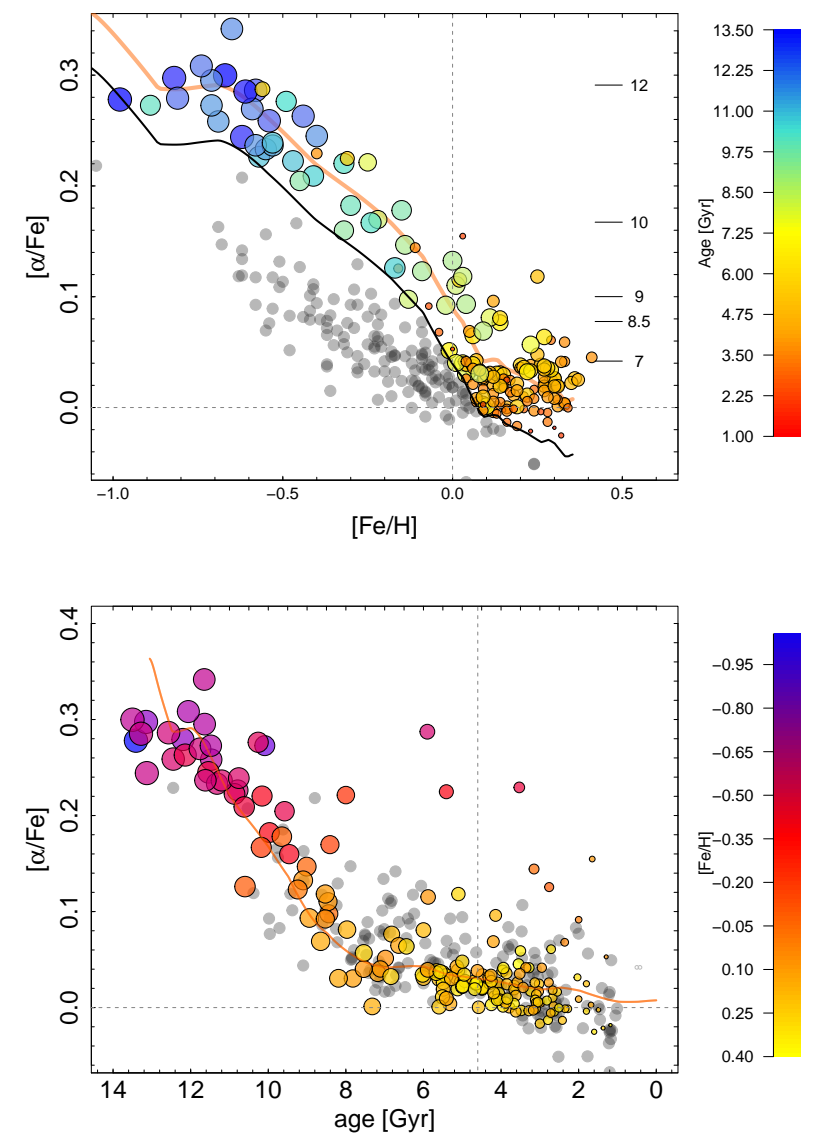

Fig. 1. Top: distribution of stars in the $[\mathrm{Fe} / \mathrm{H}]-[\alpha / \mathrm{Fe}]$ plane. The color and size of the symbols indicate the age of the stars, as shown by the vertical colorbar on the right of the panel. The orange curve is our model for the inner disk (Haywood et al. 2018; Snaith et al. 2015). Colored points are selected to be above the model curve shifted vertically by -0.05 dex. The ticks and numbers on the right side of the plot indicate the age of the model at different alpha-abundances. Bottom: distribution of stars in the age- $[\alpha / \mathrm{Fe}]$ plane, with stars selected as in the plot above. The color of the points now indicate the metallicity of the stars (right vertical colorbar)

Silva Aguirre et al. (2018) themselves and their Figs. 3 and 10. These plots show that, for stars older than $5 \mathrm{Gyr}$, the relative uncertainties in the ages they estimate are mostly greater than $30 \%$, which for a $10 \mathrm{Gyr}$ object is \pm 3 Gyr. This is reflected in their Fig. 10. Given these large uncertainties, any tight age$[\alpha / \mathrm{Fe}]$ relation will be hidden in the observational errors.

The orange curve in Fig. 1 represents our model describing the chemical evolution of the inner disk and bulge (see Snaith et al. 2015; Haywood et al. 2018). We select high- $\alpha$ stars in Fig. 1a by imposing that they must have an $[\alpha / \mathrm{Fe}]$ abundance higher than the model shifted by -0.05 dex. In Haywood et al. (2018), we show that the high- $\alpha$ sequence represents the evolution of the inner disk and bulge and can be described by a model where most of the gas has been accreted rapidly onto the disk. The model curve on the bottom plot shows two segments, representing the thick and thin (inner) disks (see Haywood et al. 2013, for a discussion). We showed in Haywood et al. (2016) that the two phases are separated by a quenching episode that occurred approximately 8-10 Gyr ago. The $\alpha$-rich sequence is a temporal sequence: alpha-abundance and metallicities are closely correlated with age (Haywood et al. 2013), as can be seen in the bottom plot, even though the stars in the sample originate from different radii. This is why on the high- $\alpha$ sequence, mono-abundance populations are also mono-age populations.

According to the studies of Snaith et al. (2015) and Haywood et al. (2015, 2018), this strong correlation between ages, alpha-abundances, and metallicities is due to the closedbox type evolution of the inner disk and bulge, described globally by a very homogeneous chemical evolution. It is apparent that chemical evolution proceeds along this sequence. The best evidence for this is that the low- $\alpha$ sequence stars are essentially absent from the inner disk, except at high metallicity, which represents the evolution of the inner thin disk. Low- $\alpha$ stars are seen at a radius of $R \sim 5 \mathrm{kpc}$, but this is the tail of a distribution that dominates the outer disk (see Hayden et al. 2015). This spatial dichotomy invalidates scenarios trying to explain the high$\alpha$ stars as the parent generation of low- $\alpha$ sequence stars (e.g., Schönrich \& Binney 2009; Nidever et al. 2014, their Fig. 17). The crucial point for the rest of the article, though, as argued in Haywood et al. (2015, 2016), is that the homogeneous chemical evolution during the thick disk phase implies that no inside-out or radially dependent formation occurred for this stellar population. Another important point to take from these two plots (see below) is that the thick disk phase reaches solar metallicity, as is already known (e.g., Bensby et al. 2007). The seven stars with ages between 8 and $9 \mathrm{Gyr}$ and $[\alpha / \mathrm{Fe}]>0.05$ in the top plot have a mean metallicity of $-0.015 \mathrm{dex}(1 \sigma$ dispersion of $0.10 \mathrm{dex})$. We note that this metallicity is higher than the metallicity of the oldest thin disk stars in the solar vicinity, implying that some amount of dilution must have occurred (see below).

\subsection{Outer disk sequence: a dilution sequence}

Figure 2 shows our sample sliced in different age intervals (see also Buder et al. 2019, their Fig. 22). The orange curve represents our model track describing the evolution of the inner disk and bulge, as in Fig. 1. This figure illustrates that the outer disk sequence is clearly stratified in age and metallicity, with older stars at higher alphas for a given metallicity, as is already known from the age- $[\alpha / \mathrm{Fe}]$ relation. Mono-abundance populations in this sequence are not mono-age populations because stars of a given age cover a large range in metallicity. The missing parameter needed to single out a mono-age population on the outer disk sequence is the birth radius of the stars, as we discuss below.

The sequences of coeval stars in Fig. 2 therefore suggest that the evolution is not from the most metal-poor stars, at $[\mathrm{Fe} / \mathrm{H}] \sim-0.7$, to the most metal-rich, at $[\mathrm{Fe} / \mathrm{H}] \sim+0.5$, as is known, also from the age-metallicity relation (see, e.g., Edvardsson et al. 1993; Haywood 2006; Casagrande et al. 2011). Therefore, the low- $\alpha$ sequence is not a temporal sequence: while the oldest stars are also the most metal-poor (plot a, see also Haywood et al. 2013; Buder et al. 2019), the most metal-rich are found at all ages below $8 \mathrm{Gyr}$. Instead, in the sequence of oldest stars, at ages between 9 and $8 \mathrm{Gyr}$, we see the first generation of stars that started to form at different metallicities in the thin disk, with dilution in stars with higher alphaabundances. The youngest sequence (age $<2$ Gyr, plot e) is the end point of evolutions that start from the oldest sequence (plot b). Because the dynamical properties of stars at the lowest metallicities in the solar vicinity suggest they come from the outer disk (Haywood 2008), and because APOGEE observed the same type of stars in situ in the outer disk, it follows that each sequence in Fig. 2 is also a sequence dependent on the birth radius of the stars. Interpreting the data this way suggests that the thin disk started to form stars with decreasing metallicity at increasing radius. Hence it is natural to interpret the low- $\alpha$ sequence 


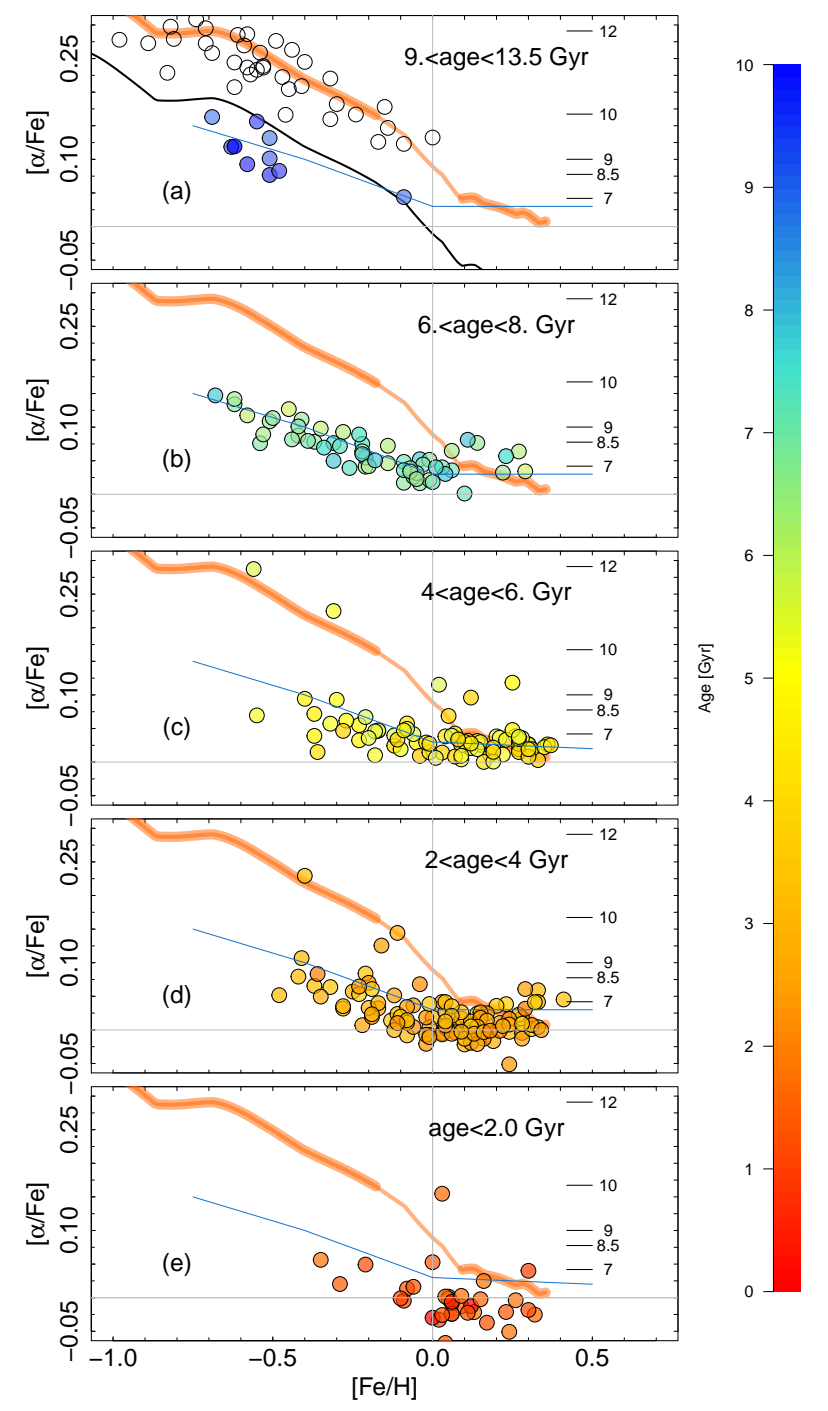

Fig. 2. Distribution of stars in the $[\mathrm{Fe} / \mathrm{H}]-[\alpha / \mathrm{Fe}]$ plane as a function of age range (top to bottom plots). The orange curve is our model for the inner disk. Ages from 12 to $7 \mathrm{Gyr}$ along this sequence are also indicated. The blue curve is the same in all plots and serves as a guide to show the shift of the distribution with age. The parallel sequences of different ages illustrate that chemical evolution in the solar vicinity (the OLR region) and beyond proceeded along evolutionary paths that link these sequences as age-metallicity sequences with the two sequences being of different age.

observed within the solar vicinity as a composite of chemical tracks, each describing an evolution at a given radius, slightly increasing in metallicity and decreasing in alpha-abundance as a function of time. We are able to observe this complexity in the solar vicinity, due to the dynamical wandering of stars born at all radii (i.e., the amplitude of their radial oscillation around their guiding centers). For the solar vicinity, such a track would show a mean evolution of metallicity as a function of age limited to a range from about -0.2 to about $0.1-0.2$, as can be measured on the age-metallicity relation (see Haywood 2006; Haywood et al. 2013). The evolution at other radii can be conceived similarly, the only difference being the initial metallicity and alpha-abundance, which at $R>6 \mathrm{kpc}$ are both a function of the distance to the Galactic center.

We can quantify how these trends change with radius and more generally we can determine the metallicity profile of the thin disk. Figure 3 shows the metallicity of the peak of the

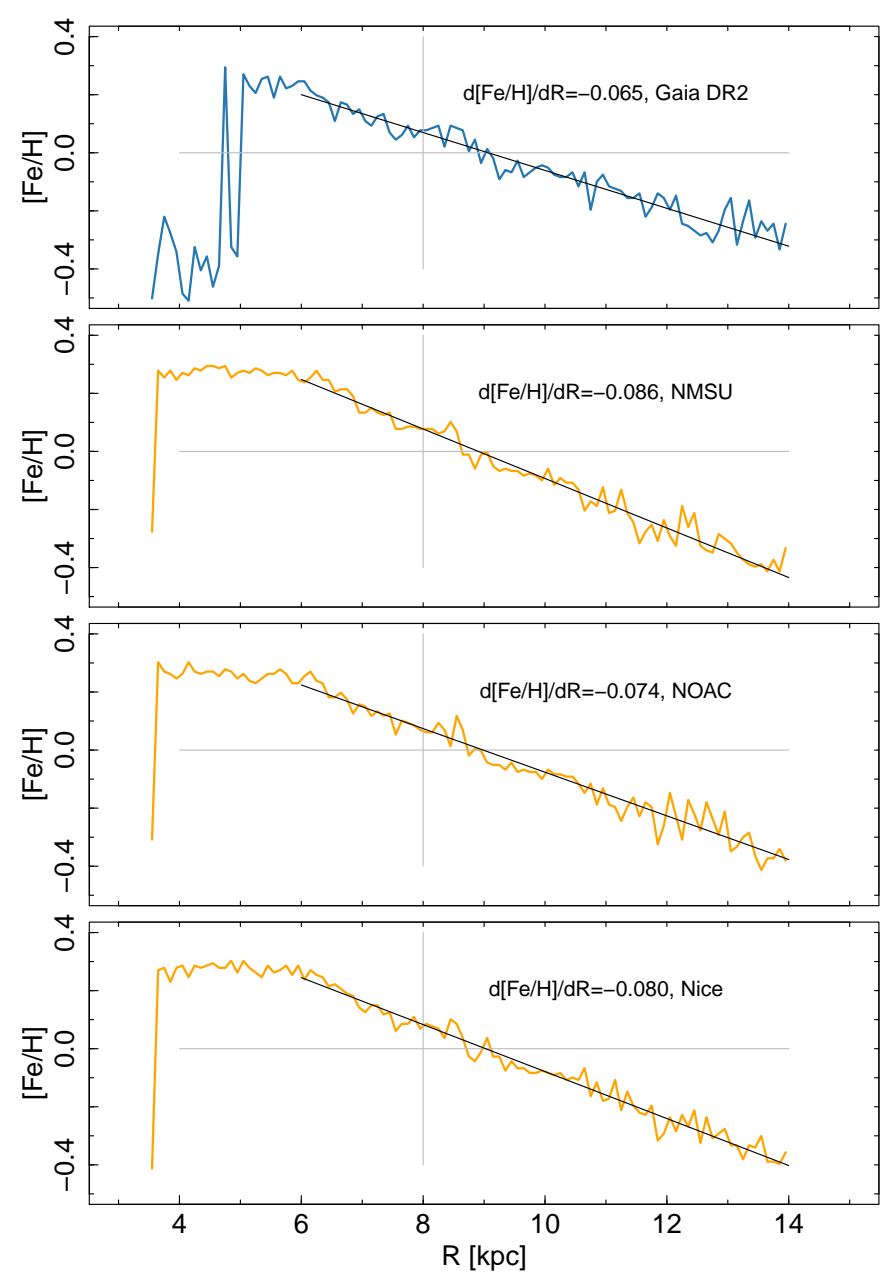

Fig. 3. Metallicity of the peak of distribution of metallicities of low- $\alpha$ stars in APOGEE as a function of $R$ for different distance estimates: Gaia (top) and then NMSU, NAOC, and Nice in the next three panels from top to bottom (as indicated, along with the radial gradient estimate, in the legend of each panel). At $R<6 \mathrm{kpc}$, the gradient is flat in all estimates (inner disk).

distribution of the low- $\alpha$ sequence as a function of $R$ using the data from the DR14 (Abolfathi et al. 2018) of the near-infrared high-resolution $(R \sim 22500)$ spectroscopic survey APOGEE (Majewski et al. 2017). The distances for the stars are taken from the parallax estimates of the Gaia DR2 (top), selecting stars with less than $20 \%$ relative error on parallaxes, and distance estimates of Holtzman et al. (2018, labeled NMSU), Wang et al. (2016, labeled NOAC) and Schultheis et al. (2014, labeled Nice). We use all stars in APOGEE DR14 that have a distance estimate, a signal-to-noise ratio higher than 50, effective temperatures lower than $5250 \mathrm{~K}$, and $\log g<3.8$.

All plots except the one with Gaia parallaxes, which lacks data for a number of APOGEE stars in zones of high extinction and crowding, show two distinct regimes: the first at $R<6 \mathrm{kpc}$ shows a flat gradient, and the second above this limit that shows a gradient between 0.065 and $0.086 \mathrm{dex} \mathrm{kpc}^{-1}$. These gradients are steeper than those found by Hayden et al. (2015), the main reason being the metallicity estimator used in each case (value of the peak in our case, and the mean in their case), and there is a difference of $\approx 0.15$ dex between the two estimates. We note that the absolute values of the metallicities may slightly overestimate the real metallicities of the population because giant stars are biased towards younger ages (Bovy et al. 2014). 


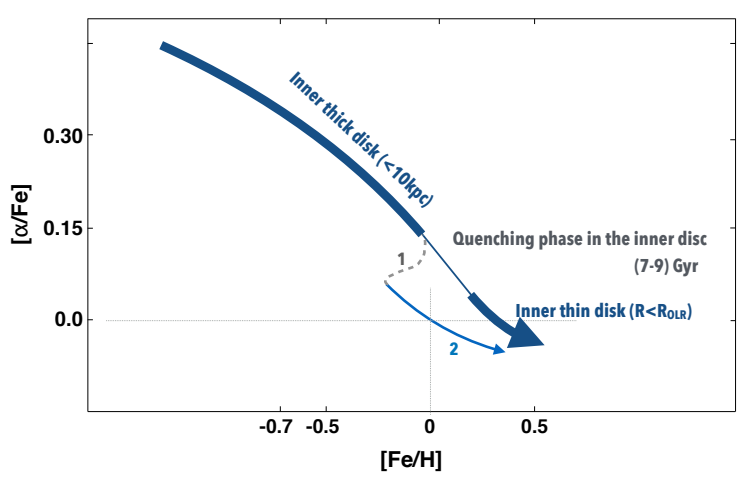

Fig. 4. Schematic representations of the chemical evolution at the solar radius in the $[\mathrm{Fe} / \mathrm{H}]-[\alpha / \mathrm{Fe}]$ plane according to the description presented in Sect. 3. The blue thin curve with the arrow (2) represents the chemical track for the solar ring evolution after an episode of dilution that occurred at the end of the thick disk formation (1).

The break in the metallicity profile of these two regions reflects the change in nature of their chemical evolution: the evolution within $R \sim 6 \mathrm{kpc}$ is closed-box-like (Haywood et al. 2018), while the evolution beyond $R \sim 6 \mathrm{kpc}$ has an evolution that was significantly impacted by dilution of the enriched gas from which it formed, as we describe below. In the outer disk, at $R>6 \mathrm{kpc}$, the gradients show at what metallicity most of the stars formed, ranging from almost +0.1 at solar radius to -0.4 at $14 \mathrm{kpc}$, illustrating the shift in the evolution as a function of radius, with chemical evolution starting at decreasing lower initial metallicity towards the outer disk, and having formed the majority of its stars also at decreasing metallicities.

\section{Solar vicinity as the prototypical example of the outer disk evolution}

\subsection{Hints from the solar vicinity chemical patterns}

There are objective facts suggesting that dilution is needed to explain the chemical evolution at the solar radius, unrelated to the G-dwarf problem. As mentioned above, there is evidence that the ISM at the end of the thick disk phase ( 8-9 Gyr ago) reached a near-solar metallicity. At the same time, we know that the solar vicinity MDF peaks at solar metallicity, with most of its stars being younger than 7-8 Gyr (the Sun being only $4.6 \mathrm{Gyr}$ old at $[\mathrm{Fe} / \mathrm{H}]=0$.). This tells us that at the solar vicinity, solar metallicity was reached two times, first about 8-9 Gyr ago at the end of the thick disk formation and then $\sim 4.5 \mathrm{Gyr}$ ago, implying a dilution episode between the two, as already suggested (e.g., Bensby et al. 2004).

If we assume that these two points are representative of the metallicity evolution at the solar ring, and given the constraints discussed above, the track followed by chemical evolution at the solar ring must have been similar to the chemical track illustrated in Fig. 4. First, the thick disk formation dominated the evolution (thick blue curve) and at the end of the thick disk phase, a dilution occurred, lowering the metallicity to $\sim-0.2 \mathrm{dex}$ (dashed curve), which is the metallicity of the oldest thin disk stars locally (Haywood 2006, 2008; Casagrande et al. 2011). We note that the solar neighborhood contains lower metallicity thin disk stars, but these are believed to most likely come from the outer disk, as shown by their kinematic and orbital properties (Haywood 2008; Bovy et al. 2012a). Then a moderate star formation rate (SFR: $2-3 M_{\odot} \mathrm{yr}^{-1}$ ) over 3-4 Gyr increased the metallicity by about +0.2 dex, reaching solar metallicity for the second time.
At the solar radius, the thick disk surface density represents about $12 \pm 4 \%$ according to Bland-Hawthorn \& Gerhard (2016). Measurements of the surface densities of the stellar monoabundance populations in Bovy et al. (2012b,a) yield higher estimates, the differences being largely a matter of how the thick disk is defined. For example, if it is defined as stars having high alpha-abundance $(>0.20$ dex on the SEGUE scale, more likely 0.15 dex with the stellar abundances used here), including stellar mono-abundance having scale heights larger than $400 \mathrm{pc}$, and in this case using estimates from Bovy et al. (2012b, as inferred from their Fig. 2), it may represent as much as 25-30\%. Adopting this upper limit, and given that Milky Way mass galaxies are observed to contain a similar mass of stars and gas at redshifts 1-1.5 (Tacconi et al. 2013; Dessauges-Zavadsky et al. 2015; Saintonge et al. 2013; Papovich et al. 2016), we can then assume that the total baryonic mass (gas+stars) at the end of the thick disk phase would represent 50-60\% of the present-day surface density at the solar ring. Thus, at the solar ring, the gas left at the end of the thick disk phase would provide an insufficient reservoir of gas to form the thin disk, and another $40-50 \%$ of gas would be necessary to reach the observed present-day mass surface density. Again, we note that we are talking here of the disk beyond $\sim 6 \mathrm{kpc}$, or beyond the possible initial position of the OLR. Inside the OLR, the description given by the closedbox model as given in Haywood et al. (2018) shows that no supplementary replenishment of gas is necessary to prolong its evolution up to the present time.

The solution to these two problems, namely the decrease in metallicity of the ISM $\sim 8-9$ Gyr ago and an insufficient amount of gas to form the thin disk at the end of the thick disk phase at the solar vicinity, implies a new supply of gas, and together provide the dilution necessary to decrease the metallicity of the ISM to $\sim-0.2$ dex after the thick disk formation, and the additional fuel to form the thin disk at the solar vicinity. The following back-of-the-envelope calculation provides a hint to the possible origin of the incoming gas. As already stated, it can be assumed that the surface density at the end of the thick disk phase was composed of half thick disk stars and half solar metallicity gas left over from the formation of the thick disk. The amount of gas acquired to form the thin disk at the end of the formation of the thick disk would then double this surface density to reach the present value. If the incoming gas that mixed with the gas left over from the thick disk formation was near-pristine, $[\mathrm{Fe} / \mathrm{H}]<-2$ dex, the metallicity of the mixture from which the first thin disk stars started to form locally would have been $[\mathrm{Fe} / \mathrm{H}]<-0.5 \mathrm{dex}$, which is at least $-0.3 \mathrm{dex}$ too low compared to what is observed. Therefore, the inflowing gas must have been significantly more metal rich.

The results of the APOGEE survey show that the metallicity of giants at the largest distances in the outer disk is about -0.5 to -0.7 . The oldest $(9-10 \mathrm{Gyr})$ low- $\alpha$ sequence stars observed in the solar vicinity (see Fig. 2), presumably of outer disk origin, have a similar metallicity. This suggests that it is also the metallicity of the gas that was in place beyond the thick disk, $R>10 \mathrm{kpc}$, and that mixed with the solar metallicity gas left over from the formation of the thick disk at its outskirts. If we fill out the $50-60 \%$ of the gas that was missing after the thick disk phase with gas at this metallicity, $-0.6 \mathrm{dex}$, the thin disk phase would start its formation with a gas mixture containing one-third of the gas coming from the thick disk at solar metallicity and two-thirds coming from the outer disk with a metallicity of -0.6. This would decrease the metallicity of the gas from about solar to $\approx-0.2$ to -0.3 dex. Thus, if the fractions we have estimated are correct, the fuel provided by the outer disk gas had the right 
metallicity to form the thin disk stars at the solar radius once it was mixed with the gas left over by the growth of the thick disk.

We note that in this scenario the decrease in the metallicity at the end of the thick disk phase that we see at the solar vicinity is due to the dilution of the ISM by gas from the outer disk (not infalling pristine gas); it is not generated by the gap in the SFH described in Haywood et al. (2016), which occurred in the inner disk. In this regard, it is also different from the results of Chiappini et al. (1997), who hypothesized that the decrease in metallicity was generated by a gap in the SFR at the solar ring combined with a continuous infall of pristine gas. Haywood et al. (2018) argue that the data for the inner disk are compatible with most of the gas being accreted very early in the Milky Way, with essentially little or no accretion after the thick disk phase within the extent of the thick disk $(R<10 \mathrm{kpc})$.

\subsection{Metal mixing in thick disks}

Is the assumption that the thick disk at $8-10 \mathrm{kpc}$ had a similar chemical evolution to that of the inner regions realistic? Observations of distant galaxies as a function of redshift show that flat metallicity gradients over a distance of $\sim 10 \mathrm{kpc}$ are common, with a small spread from galaxy to galaxy, usually a few $\pm 0.01 \mathrm{dex} \mathrm{kpc}^{-1}$ (Stott et al. 2014; Wuyts et al. 2016; Leethochawalit et al. 2016). The variation in gradients from galaxy to galaxy has been attributed to feedback strength, following results obtained by several groups simulating the formation of Milky Way-type galaxies. For example, Anglés-Alcázar et al. (2014) show that simulations with no wind usually generate steep gradients of metallicities, while Galactic outflows, by allowing the redistribution of metal-enriched gas over large scales, generate flat gradients, confirming similar results of other studies (e.g., Gibson et al. 2013; Ma et al. 2017). If the Milky Way thick disk formed from a turbulent thick layer of gas, as seems to be most probable, a flat metallicity gradient would be a natural outcome in such a model, and we do expect solar vicinity to have been enriched to the same level as the inner regions of the thick disk (i.e., not inside-out).

\subsection{When did the dilution occur and why?}

We can constrain when the dilution episode occurred within a few Gyr: it must have occurred after the thick disk reached solar metallicity, about 8-9 Gyr ago, and significantly before the birth of the Sun (at least a few Gyr) so that the ISM had time to be enriched again to its metallicity at the birth of the Sun $4.6 \mathrm{Gyr}$ ago. It can be inferred then that the dilution must have occurred 7-9 Gyr ago. The dilution could be the result of an accretion episode of gas by the Galaxy, but various arguments suggest otherwise. First, since the dilution seems to have occurred within a relatively narrow timespan, this allows us to reject the possibility that it was an effect of a long-timescale infall of gas. Second, if the material that mixed with the gas left over by the formation of the thick disk had a metallicity of $\sim-0.6$, as suggested above, it is difficult to imagine that it was accreted directly from cold flows and must have been in place before inflowing to the solar radius. The metallicity of the intergalactic medium during this epoch was likely much lower than this (Bergeron et al. 2002; Simcoe et al. 2004; Simcoe 2011). Presumably, higher angular momentum, pristine, or very low-metallicity gas was accreted in the outer parts of the Milky Way. Lehnert et al. (2014) suggest that throughout the formation of the thick disk, the star formation intensity of the Milky Way was well above the threshold for driving outflows, which likely lead to gas in the outer disk being polluted by inner disk gas, raising its metallicity to -0.6 , and fixing its $[\alpha / \mathrm{Fe}]$ at $\sim+0.15$.

Our ignorance of the gas accretion history, the metallicity of the outer disk, and the rate and fate of metals that were expelled by outflows during the formation of the thick disk means that we can only provide speculative answers as to how this initial outer disk gas composition may have been set. We can make rough estimates to show that this is not impossible, although there is no proof that it happened this way. For instance, Mackereth et al. (2017) show that the thick disk, or more precisely the high- $\alpha$ population, and the low- $\alpha$ population at $[\mathrm{Fe} / \mathrm{H}]<-0.2$ dex have approximately the same surface density locally. Assuming that they have scale lengths of $2 \mathrm{kpc}$ and $4 \mathrm{kpc}$, respectively, the outer disk at $R>10 \mathrm{kpc}$ is $\sim 10$ times less massive than the thick disk. The importance of outflows are similarly difficult to estimate, but considering that we describe the evolution of the thick disk as closely approximated by a closed-box, any metals lost via winds would have to be limited, and in particular must not be significant enough to substantially modify the overall metallicity distribution of the inner disk (unlike in the model of Hartwick 1976). If we assume that no more than $5-10 \%$ of the metals in the thick disk were expelled by outflows at a metallicity between -0.6 and -0.2 dex, which corresponds to the thick disk metallicity range at the maximum of the SFR, then a fraction between $0.1 \times 0.005$ and twice this amount of the thick disk mass in metals may have polluted the outer disk. This assumes that all metals eventually rain down to the Galactic plane before significant star formation in the outer disk. Diluted by a component roughly ten times less massive than the thick disk, the metals would raise the metallicity to a value similar to what it was in the thick disk, or -0.6 dex. These estimates are clearly very rough and neglect a number of factors that may be significant. For example, the amount of gas expelled from the thick disk may have been smaller and still have provided similar enrichment if the infall of more pristine gas from the Galactic halo occurred over a long timescale, in which case the pool of gas receiving the metals would have been even smaller and thus less diluted. The gas could then fall back onto the disk through a mechanism such as Galactic fountains (Shapiro \& Field 1976; Bregman 1980; Marinacci et al. 2011; Fraternali 2017).

How and when did this gas mix with the gas left by the thick disk formation? The epoch of the formation of bars in galaxies of the mass of the Milky Way predominately occurred 9-10 Gyr ago (Sheth et al. 2008; Melvin et al. 2014). If this is also the epoch of the formation of the bar in our Galaxy, it must have been a time of rearrangement of stars and gas due to the dynamical impact of the bar potential. If the formation of the bar is at the origin of the quenching event, as suggested in Haywood et al. (2016), and studied theoretically in Khoperskov et al. (2018), it is tempting to also associate the dilution with the impact of the bar on the gas. If the quenching episode was triggered by the formation of the bar, the outer Lindblad resonance must have been in place about 9-10 Gyr ago. Because of the clear difference of chemical properties of the disk within and beyond $\sim 6 \mathrm{kpc}$ observed today (Fig. 3), the OLR could well have been established at this radius. Estimates of the current position of the OLR vary between 6-9 kpc (Dehnen 2000), 10-11 kpc (Liu et al. 2012), and $7 \mathrm{kpc}$ (Monari et al. 2017). Given that the OLR must have shifted to larger radii as the bar pattern speed decreases, an initial position at $6 \mathrm{kpc}$ is thus plausible. At that time the thick disk, with its uniform metallicity and well-mixed gas, extended to roughly $10 \mathrm{kpc}$. Inside the OLR, the gas is driven from the corotation to the OLR (Simkin et al. 1980; Byrd et al. 1994; Rautiainen \& Salo 2000), helping to maintain a zero 
gradient inherited from the thick disk formation during the thin disk phase, as observed in Fig. 3 (see next section). The action of the bar prevented the inner disk from being subsequently diluted by radial flows. This effect is crucial to the overall validity of our proposed scenario.

If the OLR was established at $\sim 6 \mathrm{kpc}$ by the formation of the bar, it may have taken $\sim 1-2$ Gyr for the inflowing, metalpoor gas, which was at the time at larger distances, to reach the OLR radius (inflowing at a few $\mathrm{km} \mathrm{s}^{-1}$ ). Beyond $\sim 6 \mathrm{kpc}$, the metal-poor gas mixed with the solar metallicity gas with resulting mean metallicity decreasing outwards. We note that recent theoretical ideas about the impact of flowing gas on the velocity dispersion in the ambient gas suggest that this is a viable mechanism for increasing the amount of turbulence in disks (Krumholz et al. 2018). While we do not yet understand the impact of this increased turbulence may have on the star formation efficiency or perhaps even in suppressing star formation in the outer disk, an increase in the level of turbulence and the gas velocity dispersion would at the very least lead to mixing between the ambient, leftover gas from the formation of the thick disk and the less enriched gas from farther out in the disk. The efficient mixing supports our picture in that it would lead to a continuity in the metallicities that is observed in the outer disk where the only dependence appears to be a radial one. After the formation of the bar and the establishment of the OLR, the bar would slow down and the OLR would be displaced to a larger radius, but we would expect its effect as a barrier maintaining the inner and outer disks to continue, as commented in Hallé et al. (2015, 2018).

\subsection{Model for the solar vicinity}

Figure 5 illustrates a model (blue curves) representing the solar vicinity chemical evolution according to the scheme presented above. The model is based on the closed-box model (shown as the orange curve on the plot) described in Snaith et al. (2015) and which was shown to be valid to describe the whole inner disk and bulge (Haywood et al. 2018). The basic ingredients of the model are given in Snaith et al. (2015), together with a description of its main assumptions. The model for the solar metallicity first follows the closed-box chemical track from early times to $9 \mathrm{Gyr}$ ago, forming the thick disk and reaching solar metallicity. An instantaneous dilution is then introduced at $9 \mathrm{Gyr}$ and the metallicity is decreased from about solar to -0.2 dex. The SFH of the model is obtained, as in Snaith et al. (2015), by fitting the age-[Si/Fe] data of solar metallicity thin disk stars. The metallicity evolution and $([\mathrm{Fe} / \mathrm{H}],[\mathrm{Si} / \mathrm{Fe}])$ distribution are shown in the first two plots of Fig. 5. The bottom plot shows the MDF for stars created in the model after $9 \mathrm{Gyr}$, or after dilution. They represent the thin disk part of the evolution. It shows a distribution very near to what is observed in the solar vicinity for stars of the thin disk (or low- $\alpha$ stars). The real MDF would also include a small percentage of stars of the thick disk. In our scenario, the relative local fraction of the two is not related by chemical evolution (only the global fraction is), but is only an effect of the relative density distribution of the thin and thick disks locally. The relative density distribution is a consequence of the formation, and subsequently of the dynamical processes that fix their scale lengths and scale heights. As can be noted, the local MDF is at a maximum near solar metallicity, where the inner disk MDF reaches a minimum due to the quenching episode that is observed on the APOGEE data (Haywood et al. 2016) and on the bulge data (Haywood et al. 2018).
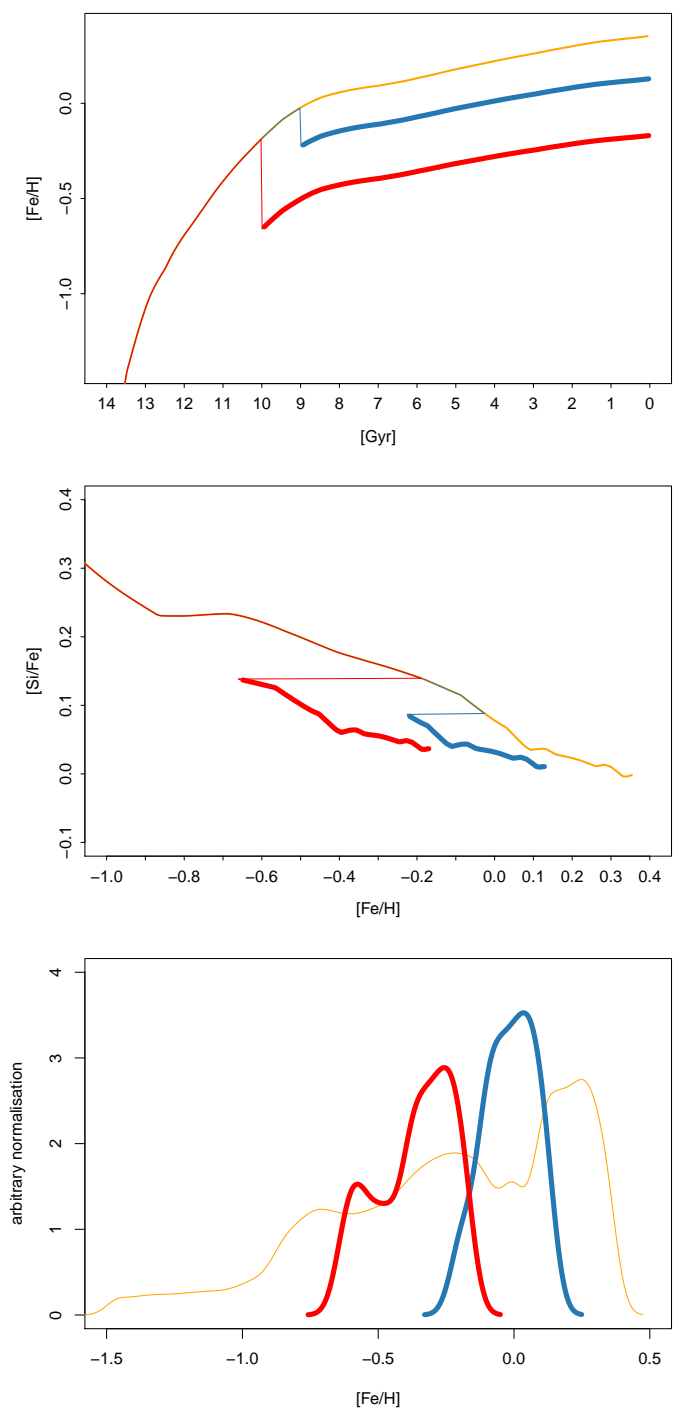

Fig. 5. Outer disk chemical evolution models according to the scheme explained in Sects. 3 and 4, describing the evolution at the solar radius (blue curves) and farther out, starting from a metallicity of -0.6 dex. In all plots, the orange curve is the closed-box chemical evolution model developed in Snaith et al. (2015) and Haywood et al. (2018). In the bottom plot, only the MDFs for stars younger than $9 \mathrm{Gyr}$ (blue curve) and 10 Gyr (red curve) are shown. Both MDFs have been smoothed by a Gaussian kernel of dispersion 0.04 dex.

\section{Generalization of our scenario to the whole outer disk}

Figure 3 shows that the solar ring is only a part of the radial metallicity profile which remains similar with a decreasing metallicity down to the limit of the sampling provided by the APOGEE data. It is therefore tempting to suggest that the scheme designed for the solar vicinity could be extended to larger distances from the Galactic center (but also to smaller distances as the plot shows that the solar ring, at $8 \mathrm{kpc}$, does not lie on the border of the inner disk).

\subsection{Models for the outer disk}

The previous section describes a two-step process. First, we imagine that the pristine gas in the outer regions of the Milky Way disk is polluted by the outflows generated during the most intense phase of the formation of the thick disk (from about 10 
to $12 \mathrm{Gyr}$ ago), raising its metallicity to about -0.6 dex. Second, at the fringe of the thick disk, this gas then mixes with the solar metallicity gas left at the end of the formation of the thick disk, as described for the solar ring in the previous section, increasing its metallicity still further relative to the outer disk. The validity of this scenario concerning the energetics of the outflows, angular momentum of the inflowing gas has to be worked out, but is clearly beyond the scope of the present work where, for the sake of clarity, we concentrate solely on the chemical evolution of the outer disk.

At the solar ring, we see that the gas from the outer disk diluted the ISM left over by formation of the thick disk (from about 0 to -0.2 dex) and provided the necessary and sufficient additional gas supply to form the thin disk, as observed in the solar vicinity. How and why the dilution occurred must be determined, but the scheme designed for the solar vicinity can be generalized assuming that at larger $R$, the relative ratio of enriched gas from the thick disk and the more metal-poor gas from the outer disk decreases, leaving a mixture of decreasing metallicity to fuel star formation. As discussed in Sect. 2, the evolution at a given radius can then be thought of as parallel sequences mirroring the evolution of that of the solar vicinity, but starting at an initial, lower metallicity. The decrease of this initial metallicity with $R$ is also reflected in the metallicity gradient of Fig. 3.

Figure 5 shows a model (red curves) where the formation of the thin disk starts slightly earlier, or $10 \mathrm{Gyr}$, and from a metallicity of -0.6 dex. This model could represent the most distant disk stars observed by APOGEE, at $\sim 14-15 \mathrm{kpc}$ from the Galactic center. The model is built in the same way as the solar vicinity model, except that because it is several kpc from the edge of the thick disk we view the metallicity as being mainly the result of the mixture of pristine gas accreted from the halo and enriched gas ejected from the thick disk, i.e., the first step mentioned at the beginning of this subsection. We note that the initial abundances of the model are fabricated this way, but it must be clear that the chemical track of the model in the first Gyr corresponds to stars formed in the thick disk. It is the formation and evolution of the thick disk that provides chemical enrichment to the outer disk via outflows, with roughly the metal budget discussed in the previous section, but that we do not expect a significant number of stars of the thick disk to have formed in the outer disk.

The resulting chemical tracks are visible in the first two plots of Fig. 5, while the third plot shows the MDF. Because the model is constrained to fit the age- $[\mathrm{Si} / \mathrm{Fe}]$ relation of Haywood et al. (2013), it must follow the knee that is visible in this relation, and which can only be fitted by lowering the SFR at about $9 \mathrm{Gyr}$, producing the dip that appears in the MDF at about -0.5 . The two models presented in Fig. 5 are representative of the evolution at two different radii in the outer disk. The only difference between the two is the initial chemical abundances from which each model starts to evolve. These two examples give us the premise from which we can conceive the evolution of the entire outer disk from $R \sim 6$ to $15 \mathrm{kpc}$, and understand the evolution underlying the classical chemical abundance plots observed in the solar vicinity.

\subsection{Sketch of the chemical trends}

The previous models are generalized in the form of three different plots sketching this evolution in the $[\mathrm{Fe} / \mathrm{H}]-[\alpha / \mathrm{Fe}]$, age$[\mathrm{Fe} / \mathrm{H}]$, and age- $[\alpha / \mathrm{Fe}]$ planes (see Fig. 6); they were made to understand how these evolutions, generalized to the whole disk, can give rise to the chemical patterns that we see in the solar vicinity. We now describe each of these plots and how they represent a generalization of the scheme that is appropriate for the solar circle.

- The evolution of the thin disk is sketched in the $[\alpha / \mathrm{Fe}]-$ $[\mathrm{Fe} / \mathrm{H}]$ plane in Fig. 6 (top), represented by a continuous series of tracks, with about $\sim 0.3$ dex increase in metallicity and about 0.1 dex decrease in $[\alpha / \mathrm{Fe}]$. The top thick blue curve represents the evolution of the inner disk, with the thick disk phase, then the quenching episode (thin blue curve), then the evolution of the thin inner disk. It shows a continuity in the evolution of the thick and the thin disks. This evolution can be reproduced by a chemical evolution model with no dilution, e.g., by a closedbox model with a two-phase SFH (see Haywood et al. 2018). However, in the outer disks, the situation is different due to the dilution, and to the likely low star formation efficiency and lower star formation rate. The lower star formation rate will produce fewer metals, and the observed lower star formation efficiency in outer disks (Bigiel et al. 2010) also suggests that the production of metals will be less efficient in enriching the ISM because of its proportionally larger gas fraction.

Due to low star formation rates and efficiencies in the outer disk, we would expect the chemical evolution tracks to have a smaller range in both $[\alpha / \mathrm{Fe}]$ and $[\mathrm{Fe} / \mathrm{H}]$. Each colored thin line represents tracks of the evolution of the outer disk at a given Galactocentric distance, starting at increasing dilution (decreasing metallicity) with increasing radii. Except for the inner thin disk track, which is the continuation of the thick disk track with no dilution, the other tracks are thus not connected to the upper $\alpha$-rich sequence, although there is possibly an indirect dependence through outflows, as discussed in the previous section. We hypothesized that in fact the initial metallicity and alphaenrichment of the outer disk comes from metals formed during the thick disk phase that polluted it (see Sect. 6). We note that this is different from studies assuming a direct jump between the two sequences in one continuous chemical evolution (see, e.g., Schönrich \& Binney 2009).

- The middle panel of Fig. 6 sketches the age-metallicity relation underlying the chemical patterns. Again, the thick blue curve represents the evolution of the inner disk (roughly within $6 \mathrm{kpc}$ ) and the thin blue line shows that the evolution of the inner disk is the continuity in the chemical enrichment of the thick disk after the quenching episode. Beyond this limit $(R>6 \mathrm{kpc})$, age-metallicity relations are diluted with respect to the inner disk evolution. Hence, at a given age the inner disk evolution is always the most metal rich of any of the sequences. In Haywood et al. (2018), we predict that the age-chemical abundance relations of the inner disk should be very tight. Before the quenching phase, the main driver of the chemical evolution of the Milky Way is the formation of the thick disk. All the curves below the inner thin disk curve represent the evolution at different radii, starting at the solar circle and progressing systematically outwards. It should be noted that the solar vicinity track is not in continuity with the thick disk evolution. As explained in Sect. 3 , the solar circle was probably diluted by $\sim 0.2$ dex. The most metal-poor objects in the outer disk are apparently as old as 9-10 Gyr (see Fig. 2, plot a); the tracks at larger radii start at progressively older ages.

- The bottom panel of Fig. 6 sketches the corresponding age$[\alpha / \mathrm{Fe}]$ relation(s). Here again, the inner disk relation is shown as the thick blue curve. Since this is a closed-box with homogeneous evolution, we expect and measured a very tight chemical evolution, as is seen in particular on the thick disk part of the relation. The outer disk evolutionary segments are parallel to the inner thin disk track, having only slightly higher alpha-abundances (Fig. 2) and, for the evolutionary track at the 

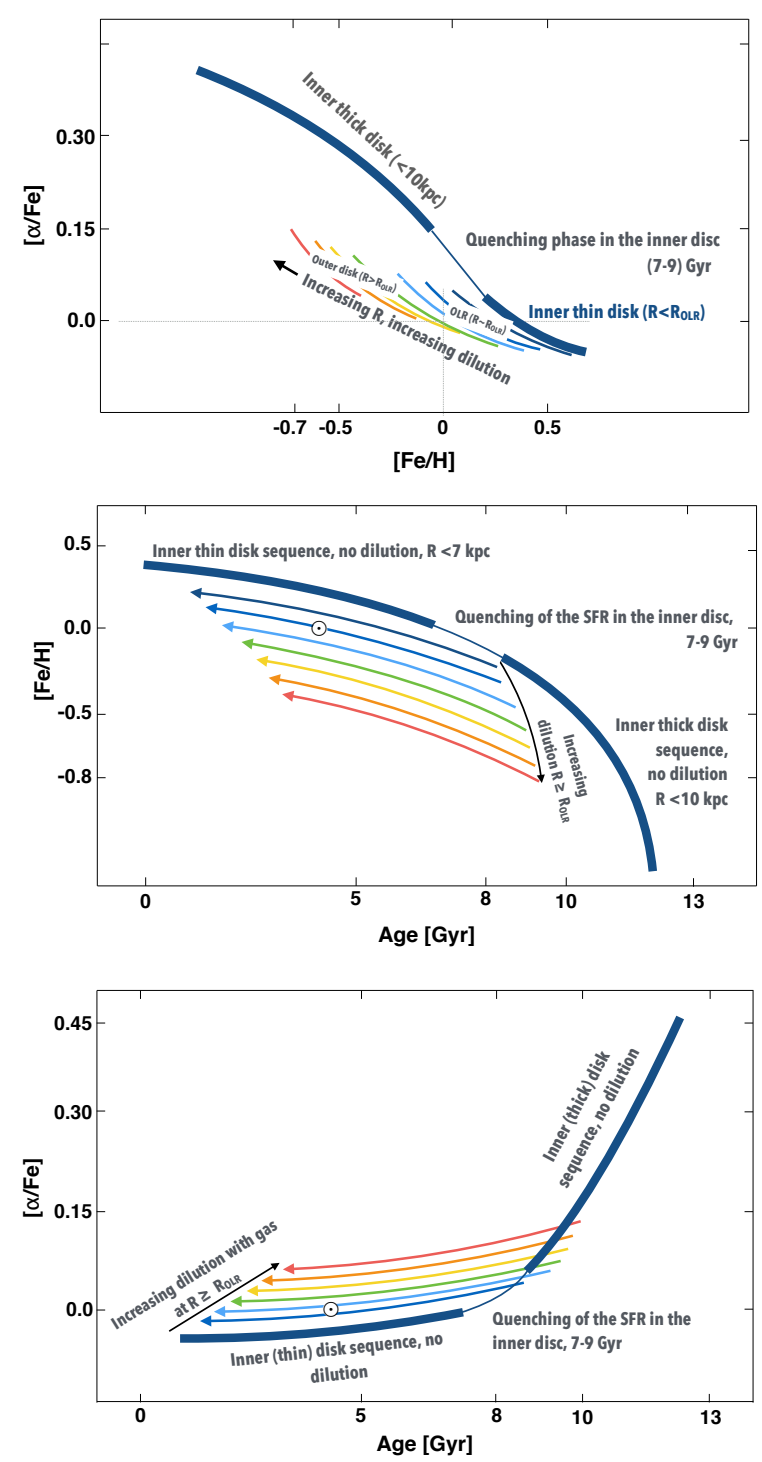

Fig. 6. Schematic representations of the $[\mathrm{Fe} / \mathrm{H}]-[\alpha / \mathrm{Fe}]$, age-metallicity, and age- $[\alpha / \mathrm{Fe}]$ relations according to the analysis presented in Sects. 3 and 4 . In all the plots, the thick curve represents the evolution of the inner disk, which is described by a closed-box model (Haywood et al 2018) with a two-phase SFH separated by a quenching episode (thinner segment along this thick curve). We expect the evolution of the inner disk to have a tight, well-defined age-metallicity relation (see Haywood et al. 2018). At a given epoch, the inner disk, $R \lesssim 6 \mathrm{kpc}$, is always the point of maximum metallicity reached by the Milky Way. The thinner, colored tracks describe the evolution of the outer disk at different radii and the initial dilution increasing with $R$. While the dilution seems to have occurred after the quenching phase at the solar radius, it may have been at earlier times in the outer disk.

largest radius (red curve), started forming stars up to 9-10 Gyr ago.

In these plots, the red sequence, which corresponds to the evolution of the far outer disk, the initial metallicity may have been set by the metals ejected by ouflows at the peak of the star formation rate during the thick disk phase, while at closer distances to the center of the Milky Way, the initial metallicity of the gas may have also been contaminated by the highly enriched gas at the end of the thick disk phase.

In the scenario we have outlined, what we are observing in the stars that lie at the solar circle is really the superposition of two different evolutionary sequences. The first is the evolution of the inner disk (thick blue curves), which is simple, continuous, and homogeneous over the scale of the whole inner disk $(R<6 \mathrm{kpc})$. The second is the evolution of the outer disk, which, due to dilution, sets initial conditions when the star formation commenced and is a function of distance to the Galactic center. In other words, it is only in the outer disk where the chemical evolution is distinctly a function of rings of constant radius. This is the regime where the chemical evolution of the Milky Way is classically modeled, i.e., as a set of independent rings.

\subsection{Global scenario}

We now summarize the various events that led to the two-phase formation of the disk. The following puts together results from various studies to try to explain the characteristics of the evolution of the disk in toto.

(1) The thick disk formed within 3-4 Gyr from 9 to $13 \mathrm{Gyr}$, in a starburst phase with a SFR reaching $\sim 12 M_{\odot} \mathrm{yr}^{-1}$ in the inner parts $(R<10 \mathrm{kpc})$ of the disk. Feedback and turbulence from the star formation activity homogenized metals in the thick disk ISM, producing a flat metallicity gradient. The most intense phase, over the age range $\sim 10-12 \mathrm{Gyr}$, generated metal outflows which polluted the outer disk, raising the metallicity at $\sim-0.6$ dex.

(2) The velocity dispersion in the gaseous turbulent disk started to decrease early, as implied from the observed correlation between the age and stellar velocity dispersion, where the vertical velocity dispersion decreases from more than $40 \mathrm{~km} \mathrm{~s}^{-1}$ at $\sim 12 \mathrm{Gyr}$ to about $30 \mathrm{~km} \mathrm{~s}^{-1}$ at $10 \mathrm{Gyr}$ (see Haywood et al. 2013). In these conditions, in a less turbulent disk, the bar started to form at an age $\sim 10 \mathrm{Gyr}$, quenched the SFR activity within the corotation region within $\sim 1 \mathrm{Gyr}$, marking the end of the thick disk formation. The formation of the OLR at $R \sim 6 \mathrm{kpc}$ isolated the inner disk from the outside. Beyond the OLR, the enriched gas $([\mathrm{Fe} / \mathrm{H}] \sim 0$ dex $)$ remaining from the thick disk formation mixed with more metal-poor gas $([\mathrm{Fe} / \mathrm{H}] \sim-0.6 \mathrm{dex})$ of the outer disk, establishing a gradient function of the fraction of the metalrich and metal-poor gas.

(3) In the inner disk, within the OLR, chemical evolution proceeded unabated after temporarily quenching, continuously processing the gas remaining from the formation of the thick disk. Haywood et al. (2018) showed that this evolution can be described with a model with no dilution, closely approximated for the last $12 \mathrm{Gyr}$ (metallicity above $-0.7 \mathrm{dex}$ ) by a closed-box model with a break in the star formation between 7 and $9 \mathrm{Gyr}$ (the quenching event).

(4) In the outer disk $(R>6 \mathrm{kpc})$, chemical evolution continued after the formation of the thick disk from increasingly lower metallicity gas at larger $R$. The gradient is a result of the mixing of the gas polluted in the outer disk with the gas remaining after the thick disk formation. The steep gradient observed up to the radial extent probed in the APOGEE survey $(R \sim 15 \mathrm{kpc})$ shows that the outer disk can be described as a series of parallel evolutions that evolved relatively separated from each other.

\section{The Sun as an outer disk star}

We now discuss whether the Sun has the characteristics of an inner or outer disk star. Specifically, in this distinction, we mean whether or not it formed out of gas that was diluted, which we argue can explain the chemical trends within and beyond $R \sim$ $6 \mathrm{kpc}$.

The Sun is offset compared to the evolution of the inner disk in two aspects, in $[\alpha / \mathrm{Fe}]$ abundance and in metallicity. This is 
illustrated by Fig. 3 for metallicity, and also in Fig. 7, which shows the $[\mathrm{Fe} / \mathrm{H}]-[\alpha / \mathrm{Fe}]$ distributions of stars in the APOGEE survey in three different distance intervals, from 5.5 to $10.5 \mathrm{kpc}$, with density contours, compared to the position of the Sun. Inner disk stars at solar metallicity have $[\alpha / \mathrm{Fe}]$ slightly above $0.1 \mathrm{dex}$ in APOGEE, as shown on Fig. 7. Because $[\alpha / \mathrm{Fe}]$ is well correlated with age, it means that the Sun is too young by a few Gyr compared to inner disk stars of the same metallicity. Stars on the high- $\alpha$ sequence at solar metallicity have ages of $\sim 9$ Gyr, meaning that the Sun is offset by at least 4 Gyr compared to stars that evolved within the inner disk. Figure 7 a shows that the peak of the low- $\alpha$, inner disk stars (at $[\mathrm{Fe} / \mathrm{H}] \sim+0.3$ and $[\alpha / \mathrm{Fe}] \sim 0$ ) is also separated from solar metallicity by almost 0.3 dex. Figure 3 is also a direct indication of the radius at which stars of solar metallicity are the most common; this is near $9 \mathrm{kpc}$ from the Galactic center.

The whole argument about the Sun having migrated from inner regions is based on the fact that it would be offset in its chemical properties compared to the population of the solar vicinity, more akin to the chemistry observed in inner disk stars. Figure 7 refutes this, and it is certainly an outlier to the inner disk chemistry. If we are to follow strictly the indication provided by Fig. 3, then the Sun has a higher probability of originating from $\sim 8.9 \mathrm{kpc}$, being slightly offset (by $0.07 \mathrm{dex}$ ) in metallicity compared to populations at $R=8 \mathrm{kpc}$.

Our position is supported by Martínez-Barbosa et al. (2015) who found, by integrating the orbit of the Sun backwards, that for all their assumed bar+spirals potentials, the origin of the Sun is always in the outer disk, sometimes as far as $11 \mathrm{kpc}$ from the Galactic center. In the case where Martínez-Barbosa et al. (2015) assume a bar with a pattern speed of $42 \mathrm{~km} \mathrm{~s}^{-1} \mathrm{kpc}^{-1}$ (OLR of the bar between 9 and $10 \mathrm{kpc}$, see their Fig. 4, which could be the position of the OLR today, see references given above) and weak spiral arms, they find that the Sun could, at most, have migrated from the outer disk by about $-0.83 \mathrm{kpc}$, which means, assuming $R_{\odot}=8 \mathrm{kpc}$, that the Sun originated from $R=8.83 \mathrm{kpc}$. This is in excellent agreement with the estimate given by the metallicity gradient above. In all the cases they considered, Martínez-Barbosa et al. (2015) found that the Sun migrated from the outer disk by small distances. This points to the result that, both for its chemistry and its dynamics, the Sun is not an inner disk object, at variance with what has been found in the last 20 years (Wielen et al. 1996; Minchev et al. 2013; Kubryk et al. 2015; Frankel et al. 2018). On the contrary, we would argue that the distributions studied here show that the Sun is much more compatible with chemical evolution with dilution, which we believe characterizes the evolution beyond $\sim 6 \mathrm{kpc}$. We conclude the Sun is an outer disk star, and has a higher probability of originating from $R>8 \mathrm{kpc}$ than the contrary.

\section{Discussion}

\subsection{Previous interpretations}

The picture we propose is different from the standard gas infall schemes in the following ways:

Thick disk growth. The initial growth of the metal content of the ISM is explained by a massive population of stars which we associate with the thick disk. Even though this population is only $15-25 \%$ of the local surface density, it represents about half the overall stellar mass of the Milky Way (see Snaith et al. 2015; Haywood et al. 2016). We argue that the high level of turbulence and feedback in the ISM at the epoch of thick disk formation allowed the outskirts of the thick disk to have a chemical
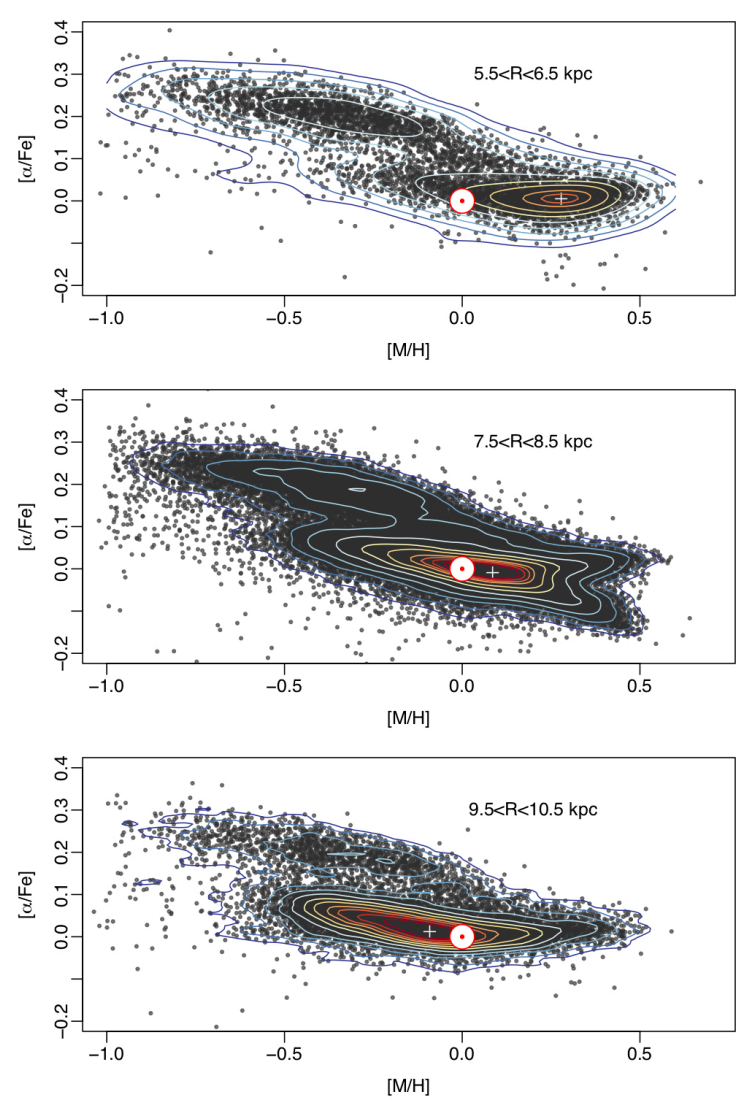

Fig. 7. $[\mathrm{M} / \mathrm{H}]-[\alpha / \mathrm{Fe}]$ distributions of stars in different distance intervals, $5.5-6.5,7.5-8.5$ and $9.5-10.5 \mathrm{kpc}$ from the Galactic center. These plots illustrate that the region where the probability of finding a solar metallicity and solar alpha-abundance star is highest beyond the solar orbit, at $R=8.89 \mathrm{kpc}$. Arguing from chemical offsets that the Sun comes from the inner disk is not supported by the APOGEE survey.

evolution similar to the inner regions. This position is supported by both the observation of the Milky Way's metallicity gradient, the gradient of galaxies at the epoch of thick disk formation, and in galaxy simulations. Hence, the disk at the Sun radius was enriched by a massive stellar population of $\sim 2.10^{10} M_{\odot}$. In this scenario, the thick disk is not formed inside-out, and the long timescale, radially dependent accretion of gas is not necessary; the chemical evolution is described well by a closed-box (with some specific SFH, see Snaith et al. 2015; Haywood et al. 2018). In inside-out scenarios, the enrichment occurs independently in rings at a particular radii: the outer regions have low gas surface densities and a slower enrichment, thus metallicities reached at the solar ring at the end of the thick disk phase are low (usually in the range $-1.0<[\mathrm{Fe} / \mathrm{H}]<-0.5 \mathrm{dex}$ ). This obviously is insufficient to provide the level of enrichment observed (see, e.g., Chiappini et al. 1997; Colavitti et al. 2009; Minchev et al. 2013). In models including radial migration, thick disk stars formed in the inner galaxy (and therefore more metal rich) are allowed to reach the solar circle. For instance, in Kubryk et al. (2015), the thick disk at the end of its evolution has a metallicity of $\sim-0.8 \mathrm{dex}$ at the solar radius, but solar metallicity thick disk stars are allowed to migrate to solar vicinity. These models, however, predict a spread in metallicity and $[\alpha / \mathrm{Fe}]$ abundance of about $0.2 \mathrm{dex}$ at the end of the thick disk phase. This is excluded by observations (Fig. 1, bottom plot). The combination of inside-out scenario and radial migration is simply not compatible with the observations (see Haywood et al. 2015 for 
more details and other arguments against an inside-out thick disk formation).

Pre-enrichment and dilution. The second phase explains the formation of the outer thin $\operatorname{disk}(R>6 \mathrm{kpc})$ to which our solar neighborhood belongs: an inflow of gas dilutes the ISM left by the thick disk phase to a metallicity compatible with the metallicity of the oldest thin disk stars (about -0.2 dex). The inflow of gas actually plays the opposite role in standard chemical evolution models, where the slow infall is used to limit as much as possible the dilution of metals.

Thus, in our scenario, the inflow of gas is not invoked to control the width of the MDF, although it contributes to determining the metallicity of the gas from which thin disk stars were formed, setting the initial metallicity of the outer thin disk decreasing with radius.

Pre-enrichment of the disk (or prompt initial enrichment, PIE) has been proposed in the past whereby top-heavy IMF provides enough metals from massive stars to solve the G-dwarf problem (see Truran \& Cameron 1971). A model with thick disk pre-enrichment has also been proposed by Gilmore \& Wyse (1986), who derived a mass ratio between the two disks of $1 / 4$, which was sufficient assuming that the thick disk would preenrich the gas to $[\mathrm{Fe} / \mathrm{H}] \sim-0.6$. However, this is no longer compatible with the solar metallicity of the youngest thick disk stars, and pre-enrichment to $[\mathrm{Fe} / \mathrm{H}]=0$, as is now required, would not be possible to reach if the thick-to-thin disk mass ratio was only $1 / 4$.

In 2001, Pagel proposed an interesting interpretation of the then recent discovery of the two separate sequences of the thin and thick disks in $[\mathrm{Fe} / \mathrm{H}]-[\alpha / \mathrm{Fe}]$. He pointed out that a combination of pre-enrichment by the thick disk and inflow at the beginning of the thin disk phase was necessary to explain the thin disk at the solar vicinity, and his Fig. 3 foreshadows our Fig. 6.

Haywood (2001) found that the solar vicinity data could be compatible with a closed-box model, provided that the thick disk contribution perpendicular to the Galactic plane was taken into account, which is usually not done (Sommer-Larsen 1991). The closed-box model envisaged in Haywood (2001) was similar to the one developed in Snaith et al. (2015), except that a constant SFR was assumed, producing a less significant metal-poor star tail than the bimodal MDF now observed in the inner disk, and which requires a more active SFR in the first Gyr, during the thick disk phase. Observations at that time did not show that the solar vicinity had peculiar chemical trends and that the thick disk had reached solar vicinity. Hence, the models could be fitted to the local MDF (with scale height corrections), but would not be adequate to describe the inner disk MDF that we now know due to the APOGEE survey.

Origin of the low- $\alpha$ sequence. Nidever et al. (2014) discuss the possibility that the low- $\alpha$ sequence could result from an evolution at low star formation efficiency and significant outflows occurring in the outer disk. Their Fig. 16 reproduces two such possible evolutionary tracks. A single such sequence would not be able to reproduce the complicated age-chemical structure of Fig. 2, but a series of them, produced by varying the star formation efficiency, could; however, this option presents two inconveniences. First, as noted by Nidever et al. (2014), the progenitor stars of the low- $\alpha$ sequence are not seen in the outer disk. These would not be expected to exist only at metallicities lower than the tail of the low- $\alpha$ sequence (or $[\mathrm{Fe} / \mathrm{H}]<-0.6$ ), but since the oldest stars of the low- $\alpha$ sequence cover the whole range of metallicities, we would expect these progenitor stars to be present at all metallicities. They are not observed. Second, the solar vicin- ity data analyzed here demonstrate that the initial metallicity of the thin disk at the solar radius was set by dilution. Given the continuity observed on the gradient of Fig. 3, it is difficult to think that this scheme would be valid only at the solar radius. We note, however, that an evolution at low star formation efficiency, as described in Nidever et al. (2014), arising from more pristine gas, unpolluted by thick disk metals could have occurred at larger distances than those probed by APOGEE.

\subsection{What does the G-dwarf distribution tells us?}

What is the meaning of the G-dwarf metallicity distribution, as a constraint for chemical evolution, in this new context? The stars that comprise the local MDF are mostly younger than 7 Gyr (80\% in our sample) and have metallicities above -0.2 dex $(73 \%)$. These stars were born from a mixture that can only be found approximately at the solar ring. In our scenario, this mixture was made from inner disk gas which was enriched through a closed-box-type evolution and outer disk gas also pre-enriched to $\sim-0.6$ dex, as described in Sects. 3 and 4.

In infall models, the width of the local MDF is used to constrain the infall timescale at the solar radius. The wider the MDF, the smaller the accretion timescale. In our scenario, the gaseous mixture from which these stars were born was in place before their formation, the necessary enrichment being provided by the formation and evolution of the thick disk. The $\mathrm{G}$ dwarfs responsible for the enrichment seen at the solar vicinity are therefore not missing, they are only not present at the solar vicinity in proportion relative to their effect on chemical evolution, because chemical evolution cannot be modeled as a strictly local process. In our view, the width of the local MDF is therefore entirely determined by an initial enrichment that was set by a global process in the Milky Way (the formation of the thick disk) and subsequent SFH. It is not a measure of the infall timescale at the solar radius.

\section{3. "The fault, dear Brutus, is not in our stars..."}

It is the mixing of stars in the disk that allows us to sample stars at solar radius that dominate at other radii and which, together with the extension of spectroscopic surveys well beyond the solar radius, allow for new insights into the chemical patterns arising from the chemical evolution of the disk. In turn, how does this mixing affect our conclusions? Mixing arises from the secular increase in the random motion of stars and their kinetic energy and/or from a change in their angular momenta, often dubbed blurring and churning in the literature. Blurring, by increasing the radial excursions of stars, contaminates other radii and increases the observed metallicity dispersion at a given radius. Because our measured gradient is based on the metallicity of the peak of the distribution, it is unlikely to be significantly affected by the increase in the metallicity dispersion at a given radius due to blurring, which is usually thought to be modest (see Binney 2007; Schönrich \& Binney 2009; Hayden et al. 2015). The effect of churning could be more important. As mentioned previously, the redistribution of angular momentum by the bar has the effect of moving material, both stars and gas, from the inner parts of galaxies to the OLR (stars: Halle et al. 2015, 2018; gas: Simkin et al. 1980; Rautiainen \& Salo 2000). It is not surprising, in these circumstances, that there is no metallicity gradient for stars within $6 \mathrm{kpc}$. First because the thick disk left no gradient and second because the action of the bar redistributes metals throughout the inner disk. These effects explain why so many metal-rich stars are found up to $R \sim 6 \mathrm{kpc}$. It was 
shown in Hallé et al. $(2015,2018)$ that this redistribution stops at the OLR, beyond which stars are not allowed to migrate via churning. This is also the case for the gas, which, accumulating at the OLR, has a tendency to form rings (Simkin et al. 1980; Rautiainen \& Salo 2000). Therefore, by moving the enriched gas from the corotation to the OLR, the formation of the bar may have provided fuel to form metal-rich stars even very near the solar orbit. In this respect, the redistribution of gas may be more important than radial migration of stars to explain the amount of metal-rich stars found up to $R \sim 6 \mathrm{kpc}$.

The fact that the metallicity of the thin disk shows a steep gradient beyond $6 \mathrm{kpc}$ (Fig. 3) supports the idea that the metalrich material cannot go on their guiding radius beyond the OLR in any significant number, as shown in Hallé et al. (2015), while stars that are far from their initial guiding radius are more likely to be there because of blurring effects (see Hallé et al. 2018). It is very possible, if the Milky Way bar is long-lived and therefore the OLR maintains its barrier effect (although it will shift to larger radii), that the only metal-rich fuel that has been available to the outer disk is the one provided by the formation of the thick disk at solar metallicity.

The steep gradient observed in Fig. 3 supports the idea that radial migration had at most a minor role in redistributing stars at the solar vicinity. The gradient at $R>6 \mathrm{kpc}$ shows that stars of a given metallicity are strongly dominating at the radius indicated by the gradient, and essentially only a small fraction are seen at other places in the disk of the Milky Way. This is supported by other studies of the solar vicinity. For example, the results of Hayden et al. (2018) are illustrative. Out of their original 2364 stars in their sample from the GES survey, 51 have $[\mathrm{Fe} / \mathrm{H}]>0.1$ dex and perigalacticon $>7 \mathrm{kpc}$ and are likely to be migrators (assuming that stars on the most circular orbits are more likely to migrate, and among them, stars with the most extreme metallicities) or $2 \%$ of the stars. Even so, one may find these criteria to be generous because the solar neighborhood is likely to form stars with $[\mathrm{Fe} / \mathrm{H}] \sim 0.1 \mathrm{dex}$, and because of the errors in the metallicities and the shape of the MDF, many more stars are likely to have estimated metallicities above $0.1 \mathrm{dex}$ than below 0.1 dex. Raising the limit in metallicity to $0.25 \mathrm{dex}$, Hayden et al. (2018) find seven stars in their sample that have a perigalacticon $>7 \mathrm{kpc}$, or $0.3 \%$. These are very likely to be real migrators and are important for explaining the spread in metallicity at a given radius. Nonetheless, it is a very small fraction, and it is difficult to argue on these grounds that churning has affected a significant number of stars in the solar vicinity and that it could affect our overall conclusions.

\section{Conclusions}

We find that the disk chemical evolution has followed two different paths depending on the distance from the Galactic center where the stars originated. One corresponds to the evolution of the inner disk, and is described well by a model where most of the gas was accreted early and evolved homogeneously, technically approximated by the closed-box model described in Haywood et al. (2018), with a two-phase SFH determined in Snaith et al. (2014, 2015). This evolution is valid up to $\sim 6 \mathrm{kpc}$ from the Galactic center and is what defines the inner disk. The formation of the outer disk would arise from the gas left by the formation of the thick disk at solar metallicity, mixed with more metal-poor gas, in a ratio which is function of $R$. The main points of this scenario are as follows:

- Due to the vigorous star formation during the formation and evolution of the thick disk inducing high turbulence and its concomitant strong gas phase mixing, the entire disk to $R \sim$ $10 \mathrm{kpc}$ (before the formation of the bar and the OLR at around $6 \mathrm{kpc}$ ) was enriched due to this population. Hence, regions like the solar ring, at the periphery of the thick disk, benefited from the enrichment of an entire massive population, although it represents only a small fraction of the surface density of the disk at the solar vicinity today.

- The thick disk enriched the disk to solar metallicity. An additional supply of more metal-poor gas must then have been available to dilute the ISM to $-0.2 \mathrm{dex}$ (the initial metallicity of the thin disk at the solar vicinity). Combined with the gas left by the thick disk, it provided the fuel necessary to form the thin disk.

- The gas present in the outer disk must have had a metallicity of about -0.6 dex at the time the thin disk started to form, based on what we can measure on the oldest outer thin disk stars. This gas is a good candidate for the dilution of the gas left by the thick disk phase. At the solar ring, the metallicity of the outer disk gas ( - 0.6 dex $)$ imposes that it contributed to two-thirds of the ISM present at the end of the thick disk formation. The other one-third corresponds to the gas left over from the formation of the thick disk. Although there is no clue to the origin of the chemical composition of the gas of the outer disk, a possibility is that it may have been pristine gas polluted by outflows generated during the formation of the thick disk (Haywood et al. 2013; Lehnert et al. 2014).

- We suggest that the mixing of the gas left from the thick disk formation with more pristine gas from the outer disk possibly occurred at the epoch of the formation of the bar and the establishment of the OLR at about $R \sim 6 \mathrm{kpc}$. Detailed simulations are needed to test this hypothesis.

- The decreasing fraction of gas left over from the formation of the thick disk induced a negative metallicity gradient in the disk at $R>6 \mathrm{kpc}$.

- From the chemical evolution point of view, the Sun is not an inner disk star, but is well on the path of chemical evolution with dilution. Thus, it is better described as an outer disk than an inner disk object, as is also supported by its orbital properties (see Martínez-Barbosa et al. 2015). The Sun is typical of the stars present at solar vicinity and does not seem to have any of the properties of the inner disk objects.

- If this scenario is correct, it means that the local G-dwarf metallicity distribution has no connection with the infall history of our Galaxy (but the inner disk MDF has; see Haywood et al. 2018), and therefore cannot be used as evidence of longtimescale gas accretion. The metallicity distribution of the solar vicinity is simply the result of a disk of gas pre-enriched to an initial metallicity of $-0.2 \mathrm{dex}$ and a mean SFR of about $1-3 M_{\odot} \mathrm{yr}^{-1}$, with no prolonged infall of gas.

Acknowledgements. The Agence Nationale de la Recherche (ANR) is acknowledged for its financial support through the MOD4Gaia project (ANR-15-CE310007, P. I.: P. Di Matteo), and also for providing the postdoctoral grant for Sergey Khoperskov. The authors are grateful to the referee for their comments and suggestions. Funding for the Sloan Digital Sky Survey IV has been provided by the Alfred P. Sloan Foundation, the U.S. Department of Energy Office of Science, and the Participating Institutions. SDSS acknowledges support and resources from the Center for High-Performance Computing at the University of Utah. The SDSS web site is www.sdss.org. SDSS is managed by the Astrophysical Research Consortium for the Participating Institutions of the SDSS Collaboration including the Brazilian Participation Group, the Carnegie Institution for Science, Carnegie Mellon University, the Chilean Participation Group, the French Participation Group, Harvard-Smithsonian Center for Astrophysics, Instituto de Astrofísica de Canarias, The Johns Hopkins University, Kavli Institute for the Physics and Mathematics of the Universe (IPMU)/University of Tokyo, Lawrence Berkeley National Laboratory, Leibniz Institut für Astrophysik Potsdam (AIP), Max-Planck-Institut für Astronomie (MPIA Heidelberg), Max-Planck-Institut für Astrophysik (MPA Garching), Max-Planck-Institut für 
Extraterrestrische Physik (MPE), National Astronomical Observatories of China New Mexico State University, New York University, University of Notre Dame, Observatório Nacional/CTI, The Ohio State University, Pennsylvania State University, Shanghai Astronomical Observatory, United Kingdom Participation Group, Universidad Nacional Autónoma de México, University of Arizona, University of Colorado Boulder, University of Oxford, University of Portsmouth University of Utah, University of Virginia, University of Washington, University of Wisconsin, Vanderbilt University, and Yale University.

\section{References}

Abolfathi, B., Aguado, D. S., Aguilar, G., et al. 2018, ApJS, 235, 42 Adibekyan, V. Z., Sousa, S. G., Santos, N. C., et al. 2012, A\&A, 545, A32 Agertz, O., Teyssier, R., Moore, B., et al. 2009, MNRAS, 397, 64

Anders, F., Chiappini, C., Santiago, B. X., et al. 2014, A\&A, 564, A115 Anglés-Alcázar, D., Davé, R., Özel, F., \& Oppenheimer, B. D. 2014, ApJ, 782,

Bensby, T., Feltzing, S., \& Lundström, I. 2004, A\&A, 415, 155

Bensby, T., Zenn, A. R., Oey, M. S., \& Feltzing, S. 2007, ApJ, 663, L13

Bensby, T., Alves-Brito, A., Oey, M. S., Young, D., \& Melendez, J. 2011, ApJ, 735, L46

Bensby, T., Feltzing, S., \& Oey, M. S. 2014, A\&A, 562, A71

Bergeron, J., Aracil, B., Petitjean, P., \& Pichon, C. 2002, A\&A, 396, L11

Bigiel, F., Leroy, A., Walter, F., et al. 2010, AJ, 140, 1194

Binney, J. 2007, Astrophys. Space Sci. Proc., 3, 67

Birnboim, Y., \& Dekel, A. 2003, MNRAS, 345, 349

Bland-Hawthorn, J., \& Gerhard, O. 2016, ARA\&A, 54, 529

Bovy, J., Rix, H.-W., Liu, C., et al. 2012a, ApJ, 753, 148

Bovy, J., Rix, H.-W., \& Hogg, D. W. 2012b, ApJ, 751, 131

Bovy, J., Nidever, D. L., Rix, H.-W., et al. 2014, ApJ, 790, 127

Bovy, J., Rix, H.-W., Schlafly, E. F., et al. 2016, ApJ, 823, 30

Bregman, J. N. 1980, ApJ, 236, 577

Buder, S., Lind, K., Ness, M. K., et al. 2019, A\&A, 624, A19

Byrd, G., Rautiainen, P., Salo, H., Buta, R., \& Crocher, D. A. 1994, AJ, 108, 476

Casagrande, L., Schönrich, R., Asplund, M., et al. 2011, A\&A, 530, A138

Ceverino, D., Sánchez Almeida, J., Muñoz Tuñón, C., et al. 2016, MNRAS, 457 2605

Cheng, J. Y., Rockosi, C. M., Morrison, H. L., et al. 2012, ApJ, 746, 149

Chiappini, C., Matteucci, F., \& Gratton, R. 1997, ApJ, 477, 765

Colavitti, E., Cescutti, G., Matteucci, F., \& Murante, G. 2009, A\&A, 496, 429

Cornuault, N., Lehnert, M. D., Boulanger, F., \& Guillard, P. 2018, A\&A, 610, A75

Davé, R., Finlator, K., \& Oppenheimer, B. D. 2012, MNRAS, 421, 98

Dehnen, W. 2000, AJ, 119, 800

Dekel, A., \& Birnboim, Y. 2006, MNRAS, 368, 2

Dessauges-Zavadsky, M., Zamojski, M., Schaerer, D., et al. 2015, A\&A, 577, A50

Edvardsson, B., Andersen, J., Gustafsson, B., et al. 1993, A\&A, 275, 101

Frankel, N., Rix, H.-W., Ting, Y.-S., Ness, M., \& Hogg, D. W. 2018, ApJ, 865, 96

Fraternali, F. 2017, Gas Accretion onto Galaxies (Springer), 430, 323

Genzel, R., Tacconi, L. J., Eisenhauer, F., et al. 2006, Nature, 442, 786

Genzel, R., Schreiber, N. M. F., Übler, H., et al. 2017, Nature, 543, 397

Gibson, B. K., Pilkington, K., Brook, C. B., Stinson, G. S., \& Bailin, J. 2013 A\&A, 554, A47

Gilmore, G., \& Wyse, R. F. G. 1986, Nature, 322, 806

Grisoni, V., Spitoni, E., Matteucci, F., et al. 2017, MNRAS, 472, 3637

Hallé, A., Di Matteo, P., Haywood, M., \& Combes, F. 2015, A\&A, 578, A58

Hallé, A., Di Matteo, P., Haywood, M., \& Combes, F. 2018, A\&A, 616, A86

Hartwick, F. D. A. 1976, ApJ, 209, 418

Hayden, M. R., Bovy, J., Holtzman, J. A., et al. 2015, ApJ, 808, 132

Hayden, M. R., Recio-Blanco, A., de Laverny, P., et al. 2018, A\&A, 609, A79

Haywood, M. 2001, MNRAS, 325, 1365

Haywood, M. 2006, MNRAS, 371, 1760

Haywood, M. 2008, MNRAS, 388, 1175

Haywood, M., Di Matteo, P., Lehnert, M., Katz, D., \& Gómez, A. 2013, A\&A, 560, A 109

Haywood, M., Di Matteo, P., Snaith, O., \& Lehnert, M. D. 2015, A\&A, 579, A5
Haywood, M., Lehnert, M. D., Di Matteo, P., et al. 2016, A\&A, 589, A66 Haywood, M., Di Matteo, P., Lehnert, M., et al. 2018, A\&A, 618, A78 Holtzman, J. A., Hasselquist, S., Shetrone, M., et al. 2018, AJ, 156, 125 Hopkins, P. F., Kereš, D., Oñorbe, J., et al. 2014, MNRAS, 445, 581

Kereš, D., Katz, N., Weinberg, D. H., \& Davé, R. 2005, MNRAS, 363, 2 Kereš, D., Katz, N., Fardal, M., Davé, R., \& Weinberg, D. H. 2009, MNRAS, 395,160

Khoperskov, S., Haywood, M., Di Matteo, P., Lehnert, M. D., \& Combes, F. 2018, A\&A, 609, A60

Krumholz, M. R., \& Dekel, A. 2012, ApJ, 753, 16

Krumholz, M. R., Burkhart, B., Forbes, J. C., \& Crocker, R. M. 2018, MNRAS, 477, 2716

Kubryk, M., Prantzos, N., \& Athanassoula, E. 2015, A\&A, 580, A126

Leethochawalit, N., Jones, T. A., Ellis, R. S., et al. 2016, ApJ, 820, 84

Lehnert, M. D., Di Matteo, P., Haywood, M., \& Snaith, O. N. 2014, ApJ, 789, L30

Loebman, S. R., Debattista, V. P., Nidever, D. L., et al. 2016, ApJ, 818, L6

Liu, C., Xue, X., Fang, M., et al. 2012, ApJ, 753, L24

Ma, X., Hopkins, P. F., Wetzel, A. R., et al. 2017, MNRAS, 467, 2430

Majewski, S. R., Schiavon, R. P., Frinchaboy, P. M., et al. 2017, AJ, 154, 94

Mackereth, J., Bovy, J., Schiavon, R. P., et al. 2017, MNRAS, 471, 3057

Marcon-Uchida, M. M., Matteucci, F., \& Costa, R. D. D. 2010, A\&A, 520, A35

Marinacci, F., Fraternali, F., Nipoti, C., et al. 2011, MNRAS, 415, 1534

Martínez-Barbosa, C. A., Brown, A. G. A., \& Portegies Zwart, S. 2015, MNRAS, 446, 823

Melvin, T., Masters, K., Lintott, C., et al. 2014, MNRAS, 438, 2882

Minchev, I., Chiappini, C., \& Martig, M. 2013, A\&A, 558, A9

Minchev, I., Chiappini, C., \& Martig, M. 2014, A\&A, 572, A92

Minchev, I., Anders, F., Recio-Blanco, A., et al. 2018, MNRAS, 481, 1645

Monari, G., Kawata, D., Hunt, J. A. S., \& Famaey, B. 2017, MNRAS, 466, L113

Muzzin, A., Marchesini, D., Stefanon, M., et al. 2013, ApJ, 777, 18

Nidever, D. L., Bovy, J., Bird, J. C., et al. 2014, ApJ, 796, 38

Ocvirk, P., Pichon, C., \& Teyssier, R. 2008, MNRAS, 390, 1326

Pagel, B. E. J. 2001, Cosmic Evolution (Singapore: World Scientific), 223

Pagel, B. E. J., \& Patchett, B. E. 1975, MNRAS, 172, 13

Papovich, C., Finkelstein, S. L., Ferguson, H. C., Lotz, J. M., \& Giavalisco, M. 2011, MNRAS, 412, 1123

Papovich, C., Labbé, I., Quadri, R., et al. 2015, ApJ, 803, 26

Papovich, C., Labbé, I., Glazebrook, K., et al. 2016, Nat. Astron., 1, 0003

Patel, S. G., van Dokkum, P. G., Franx, M., et al. 2013, ApJ, 766, 15

Rautiainen, P., \& Salo, H. 2000, A\&A, 362, 465

Saintonge, A., Lutz, D., Genzel, R., et al. 2013, ApJ, 778, 2

Shapiro, P. R., \& Field, G. B. 1976, ApJ, 205, 762

Schönrich, R., \& Binney, J. 2009, MNRAS, 399, 1145

Schultheis, M., Zasowski, G., Allende Prieto, C., et al. 2014, AJ, 148, 24

Sheth, K., Elmegreen, D. M., Elmegreen, B. G., et al. 2008, ApJ, 675, 1141

Silva Aguirre, V., Bojsen-Hansen, M., Slumstrup, D., et al. 2018, MNRAS, 475, 5487

Simcoe, R. A. 2011, ApJ, 738, 159

Simcoe, R. A., Sargent, W. L. W., \& Rauch, M. 2004, ApJ, 606, 92

Simkin, S. M., Su, H. J., \& Schwarz, M. P. 1980, ApJ, 237, 404

Snaith, O. N., Haywood, M., Di Matteo, P., et al. 2014, ApJ, 781, L31

Snaith, O., Haywood, M., Di Matteo, P., et al. 2015, A\&A, 578, A87

Sommer-Larsen, J. 1991, MNRAS, 249, 368

Stott, J. P., Sobral, D., Swinbank, A. M., et al. 2014, MNRAS, 443, 2695

Suess, K. A., Bezanson, R., Spilker, J. S., et al. 2017, ApJ, 846, L14

Tacconi, L. J., Neri, R., Genzel, R., et al. 2013, ApJ, 768, 74

Tillson, H., Devriendt, J., Slyz, A., Miller, L., \& Pichon, C. 2015, MNRAS, 449, 4363

Toft, S., Zabl, J., Richard, J., et al. 2017, Nature, 546, 510

Toyouchi, D., \& Chiba, M. 2018, ApJ, 855, 104

Truran, J. W., \& Cameron, A. G. W. 1971, Ap\&SS, 14, 179

van den Bergh, S. 1962, AJ, 67, 486

van Dokkum, P. G., Leja, J., Nelson, E. J., et al. 2013, ApJ, 771, L35

Wang, J., Shi, J., Pan, K., et al. 2016, MNRAS, 460, 3179

Wielen, R., Fuchs, B., \& Dettbarn, C. 1996, A\&A, 314, 438

Woods, R. M., Wadsley, J., Couchman, H. M. P., Stinson, G., \& Shen, S. 2014 , MNRAS, 442, 732

Wuyts, E., Wisnioski, E., Fossati, M., et al. 2016, ApJ, 827, 74 